\title{
Invariants in Separated Variables: Yang-Baxter, Entwining and Transfer Maps
}

\author{
Pavlos KASSOTAKIS
}

Department of Mathematics and Statistics, University of Cyprus, P.O. Box 20537, 1678 Nicosia, Cyprus

E-mail: pavlos1978@gmail.com,pkasso01@ucy.ac.cy

Received January 16, 2019, in final form June 15, 2019; Published online June 25, 2019

https://doi.org/10.3842/SIGMA.2019.048

\begin{abstract}
We present the explicit form of a family of Liouville integrable maps in 3 variables, the so-called triad family of maps and we propose a multi-field generalisation of the latter. We show that by imposing separability of variables to the invariants of this family of maps, the $H_{\mathrm{I}}, H_{\mathrm{II}}$ and $H_{\mathrm{III}}^{A}$ Yang-Baxter maps in general position of singularities emerge. Two different methods to obtain entwining Yang-Baxter maps are also presented. The outcomes of the first method are entwining maps associated with the $H_{\mathrm{I}}, H_{\mathrm{II}}$ and $H_{\mathrm{III}}^{A}$ Yang-Baxter maps, whereas by the second method we obtain non-periodic entwining maps associated with the whole $F$ and $H$-list of quadrirational Yang-Baxter maps. Finally, we show how the transfer maps associated with the $H$-list of Yang-Baxter maps can be considered as the $(k-1)$-iteration of some maps of simpler form. We refer to these maps as extended transfer maps and in turn they lead to $k$-point alternating recurrences which can be considered as alternating versions of some hierarchies of discrete Painlevé equations.
\end{abstract}

Key words: discrete integrable systems; Yang-Baxter maps; entwining maps; transfer maps

2010 Mathematics Subject Classification: 14E07; 14H70; 37K10

\section{Introduction}

The quantum Yang-Baxter equation originates from the theory of exactly solvable models in statistical mechanics $[11,73]$. It reads

$$
R_{12} R_{13} R_{23}=R_{23} R_{13} R_{12}
$$

where $R: V \otimes V \mapsto V \otimes V$ a linear operator and $R_{l m}, l \neq m \in\{1,2,3\}$ the operators that acts as $R$ on the $l$-th and $m$-th factors of the tensor product $V \otimes V \otimes V$. For the history of the latter and for the early developments on the theory see [36]. Replacing the vector space $V$ with any set $X$ and the tensor product with the cartesian product, Drinfeld [21] introduced the set theoretical version of (1.1). Solutions of the latter appeared under the name of set theoretical solutions of the quantum Yang-Baxter equation. The first instance of such solutions, appeared in $[24,65]$. The term Yang-Baxter maps was proposed by Veselov [69] as an alternative name to the Drinfeld's one. Early results on the context of Yang-Baxter maps were provided in $[1,40,57]$. In the recent years, many results arose in the interplay between studies on Yang-Baxter maps and the theory of discrete integrable systems [8, 9, 10, 12, 18, 19, 20, 31].

In [23] it was considered a special type of set theoretical solutions of the quantum YangBaxter equation, the so called non degenerate rational maps. Nowadays, this type of solutions is referred to as quadrirational Yang-Baxter maps. Note that the notion of quadrirational maps, was extended in [46] to the notion of $2^{n}$-rational maps, where highly symmetric higher dimensional maps were considered. Under the assumption of quadrirationality and modulo conjugation (see Definition 3.1), in [5,59] a list of ten families of maps was obtained. Five 
of them were given in [5], which constitute the so-called F-list of quadrirational Yang-Baxter maps and five more in [59], which constitute the so-called $H$-list of quadrirational Yang-Baxter maps. For their explicit form see Appendix A. The Yang-Baxter maps of the F-list and the $H$-list can also be obtained from some of the integrable lattice equations in the classification scheme of [4], by using the invariants of the generators of the Lie point symmetry group of the latter [60]. In the series of papers [44, 45, 56], from the Yang-Baxter maps of the F-list and of the $H$-list, integrable lattice equations and correspondences (relations) were systematically constructed. Invariant, under the maps, functions where the variables appeared in separated form, played an important role to this construction. The cornerstone of this manuscript are invariant functions where the variables appear in separated form.

In [3], it was introduced a family rational of maps in 3 variables that preserves two rational functions the so-called the triad map. The triad map serves as a generalisation of the QRT map [61] (cf. [22]). In Section 2 we present an explicit formula for Adler's triad map as well as we prove the Liouville integrability of the latter. We also propose an extension of the triad map in $k \geq 3$ number of variables. If one imposes separability to the variables of the invariants of the triad map, the $H_{\mathrm{I}}$, the $H_{\mathrm{II}}$ and the $H_{\mathrm{III}}^{A}$ Yang-Baxter maps in general positions of singularities, emerge. This is presented in Section 3 together with the explicit formulae for these maps.

In Section 4, we develop two methods to obtain non-equivalent entwining maps [51], i.e., maps $R, S, T$ that satisfy the relation

$$
R_{12} S_{13} T_{23}=T_{23} S_{13} R_{12} \text {. }
$$

The first method gives us entwining maps associated with the $H_{\mathrm{I}}, H_{\mathrm{II}}$ and the $H_{\mathrm{III}}^{A}$ members of the $H$-list of Yang-Baxter maps. The second one produces entwining maps for the whole $F$-list and the $H$-list. In this manuscript we present the entwining maps associated with the $H$-list of quadrirational Yang-Baxter maps only.

In Section 5, we re-factorise the transfer maps [69] associated with the $H$-list of Yang-Baxter maps. We show that the transfer maps coincide with the $(k-1)$-iteration of some maps of simpler form that we refer to as extended transfer maps. Moreover, we show that the extended transfer maps, after an integration followed by a change of variables, are written as $k$-point recurrences, which some of them can be considered as alternating versions of discrete Painlevé hierarchies $[16,32,57]$. In Section 6 we end this manuscript with some conclusions and perspectives.

\section{The Adler's triad family of maps}

In [3], Adler proposed the so-called triad family of maps. The triad map is a family of maps in 3 variables that consists of the composition of involutions which preserve two rational invariants of a specific form. In what follows we present the explicit form of the latter in terms of its invariants.

Consider the polynomials

$$
n^{i}=\sum_{j, k, l=0}^{1} \alpha_{j, k, l}^{i} x_{1}^{1-j} x_{2}^{1-k} x_{3}^{1-l}, \quad d^{i}=\sum_{j, k, l=0}^{1} \beta_{j, k, l}^{i} x_{1}^{1-j} x_{2}^{1-k} x_{3}^{1-l}, \quad i=1,2,
$$

where $x_{1}, x_{2}, x_{3}$ are considered as variables and $\alpha_{j, k, l}^{i}, \beta_{j, k, l}^{i}$ as parameters. We consider also 3 maps $R_{i j}, i<j, i, j \in\{1,2,3\}$. These maps can be build out of the polynomials $n^{i}, d^{i}$ and they $\operatorname{read} R_{i j}:\left(x_{1}, x_{2}, x_{3}\right) \mapsto\left(X_{1}\left(x_{1}, x_{2}, x_{3}\right), X_{2}\left(x_{1}, x_{2}, x_{3}\right), X_{3}\left(x_{1}, x_{2}, x_{3}\right)\right)$, where

$$
X_{i}=x_{i}-2 \frac{\left|\begin{array}{ll}
\mathbf{D}_{x_{i}} n^{1} \cdot d^{1} & \mathbf{D}_{x_{i}} n^{2} \cdot d^{2} \\
\mathbf{D}_{x_{j}} n^{1} \cdot d^{1} & \mathbf{D}_{x_{j}} n^{2} \cdot d^{2}
\end{array}\right|}{\left|\begin{array}{cc}
\mathbf{D}_{x_{i}} n^{1} \cdot d^{1} \\
\mathbf{D}_{x_{i}} n^{2} \cdot d^{2} \\
\partial_{x_{i}} \mathbf{D}_{x_{j}} n^{1} \cdot d^{1}+\partial_{x_{j}} \mathbf{D}_{x_{i}} n^{1} \cdot d^{1} & \partial_{x_{i}} \mathbf{D}_{x_{j}} n^{2} \cdot d^{2}+\partial_{x_{j}} \mathbf{D}_{x_{i}} n^{2} \cdot d^{2}
\end{array}\right|},
$$




$$
\begin{aligned}
& X_{j}=x_{j}+2 \frac{\left|\begin{array}{lc}
\mathbf{D}_{x_{i}} n^{1} \cdot d^{1} & \mathbf{D}_{x_{i}} n^{2} \cdot d^{2} \\
\mathbf{D}_{x_{j}} n^{1} \cdot d^{1} & \mathbf{D}_{x_{j}} n^{2} \cdot d^{2}
\end{array}\right|}{\left|\begin{array}{cc}
\mathbf{D}_{x_{j}} n^{2} \cdot d^{2} \\
\mathbf{D}_{x_{j}} n^{1} \cdot d^{1} \\
\partial_{x_{i}} \mathbf{D}_{x_{j}} n^{1} \cdot d^{1}+\partial_{x_{j}} \mathbf{D}_{x_{i}} n^{1} \cdot d^{1} & \partial_{x_{i}} \mathbf{D}_{x_{j}} n^{2} \cdot d^{2}+\partial_{x_{j}} \mathbf{D}_{x_{i}} n^{2} \cdot d^{2}
\end{array}\right|}, \\
& X_{k}=x_{k} \quad \text { for } \quad k \neq i, j \text {, }
\end{aligned}
$$

with $\partial_{z}$ we denote the partial derivative operator w.r.t. to $z$, i.e., $\partial_{z} h=\frac{\partial h}{\partial z} \cdot \mathbf{D}_{z}$ is the Hirota's bilinear operator, i.e., $\mathbf{D}_{z} h \cdot k=\left(\partial_{z} h\right) k-h \partial_{z} k$.

Proposition 2.1. The following holds:

1. Mappings $R_{i j}$ depend on 32 parameters $\alpha_{j, k, l}^{i}, \beta_{j, k, l}^{i}, i=1,2, j, k, l \in\{0,1\}$. Only 15 of them are essential.

2. The functions $H_{1}=n^{1} / d^{1}, H_{2}=n^{2} / d^{2}$ are invariant under the action of $R_{i j}$, i.e., $H_{l} \circ$ $R_{i j}=H_{l}, l=1,2$.

3. Mappings $R_{i j}$ are involutions, i.e., $R_{i j}^{2}=\mathrm{id}$.

4. Mappings $R_{i j}$ are anti-measure preserving ${ }^{1}$ with densities $m_{1}=n^{1} d^{2}, m_{2}=n^{2} d^{1}$.

5. Mappings $R_{i j}$ satisfy the relation $R_{12} R_{13} R_{23}=R_{23} R_{13} R_{12}$.

Proof. 1. The invariants $H_{1}, H_{2}$ depend on 3 variables and they include 32 parameters. Acting with a different Möbius transformation to each of the variables, 9 parameters can be removed. A Möbius transformation of an invariant remains an invariant, since we have 2 invariants, 6 more parameters can be removed. Finally, since any multiple of an invariant remains an invariant, 2 more parameters can be removed. That leaves us with $32-9-6-2=15$ essential parameters for the invariants $H_{1}, H_{2}$ and hence for the maps $R_{i j}$.

2. The functions $H_{1}=n^{1} / d^{1}, H_{2}=n^{2} / d^{2}$, reads

$$
\begin{aligned}
& H_{1}\left(x_{1}, x_{2}, x_{3}\right)=\frac{a x_{1} x_{2}+b x_{1}+c x_{2}+d}{a_{1} x_{1} x_{2}+b_{1} x_{1}+c_{1} x_{2}+d_{1}}, \\
& H_{2}\left(x_{1}, x_{2}, x_{3}\right)=\frac{k x_{1} x_{2}+l x_{1}+m x_{2}+n}{k_{1} x_{1} x_{2}+l_{1} x_{1}+m_{1} x_{2}+n_{1}},
\end{aligned}
$$

where $a, a_{1}, b, b_{1}, k, k_{1}, \ldots$ are linear functions of $x_{3}$ (note we have suppressed the dependency on $x_{3}$ of the functions $\left.H_{1}, H_{2}\right)$. From the set of equations

$$
H_{1}\left(X_{1}, X_{2}, x_{3}\right)=H_{1}\left(x_{1}, x_{2}, x_{3}\right), \quad H_{2}\left(X_{1}, X_{2}, x_{3}\right)=H_{2}\left(x_{1}, x_{2}, x_{3}\right),
$$

by eliminating $X_{2}$ or by eliminating $X_{1}$ the resulting equations respectively factorize as

$$
\left(X_{1}-x_{1}\right) A=0, \quad\left(X_{2}-x_{2}\right) B=0 .
$$

The factor $A$ is linear in $X_{1}$ and the factor $B$ is linear in $X_{2}$. By solving these equations (we omit the trivial solution $X_{1}=x_{1}, X_{2}=x_{2}$ ) we obtain

$$
\begin{aligned}
& X_{1}=\frac{\gamma_{13}^{34} x_{2}^{2}+\left(\gamma_{23}^{34}+\gamma_{14}^{34}\right) x_{2}+\gamma_{24}^{34}+\left(\gamma_{13}^{14} x_{2}^{2}+\left(\gamma_{13}^{24}+\gamma_{23}^{14}\right) x_{2}+\gamma_{23}^{24}\right) x_{1}}{\gamma_{13}^{23} x_{2}^{2}+\left(\gamma_{13}^{24}+\gamma_{14}^{23}\right) x_{2}+\gamma_{14}^{24}+\left(\gamma_{12}^{13} x_{2}^{2}+\left(\gamma_{12}^{23}+\gamma_{12}^{14}\right) x_{2}+\gamma_{12}^{24}\right) x_{1}}, \\
& X_{2}=\frac{\gamma_{12}^{24} x_{1}^{2}+\left(\gamma_{24}^{23}+\gamma_{14}^{24}\right) x_{1}+\gamma_{34}^{24}+\left(\gamma_{12}^{14} x_{1}^{2}+\left(\gamma_{12}^{34}+\gamma_{14}^{23}\right) x_{1}+\gamma_{34}^{23}\right) x_{2}}{\gamma_{23}^{12} x_{1}^{2}+\left(\gamma_{12}^{34}+\gamma_{23}^{14}\right) x_{1}+\gamma_{14}^{34}+\left(\gamma_{13}^{12} x_{1}^{2}+\left(\gamma_{23}^{13}+\gamma_{13}^{14}\right) x_{1}+\gamma_{13}^{34}\right) x_{2}},
\end{aligned}
$$

\footnotetext{
${ }^{1} \mathrm{~A}$ map $\phi:(x, y) \mapsto(X, Y)$ is called measure preserving map with density $m(x, y)$, if its Jacobian determinant $\frac{\partial(X, Y)}{\partial(x, y)}$ equals to $\frac{m(X, Y)}{m(x, y)}$. If the Jacobian determinant of the map $\phi$ equals to $-\frac{m(X, Y)}{m(x, y)}$, then the map $\phi$ is called anti-measure preserving map with density $m(x, y)$.
} 
where $\gamma_{i j}^{k l}:=\left|\begin{array}{ll}u_{i j} & u_{k l} \\ v_{i j} & v_{k l}\end{array}\right|$, with $u_{i j}$ the determinants of a matrix generated by the $i^{\text {th }}$ and $j^{\text {th }}$ column of the matrix

$$
u=\left(\begin{array}{cccc}
a & b & c & d \\
a_{1} & b_{1} & c_{1} & d_{1}
\end{array}\right)
$$

and $v_{k l}$ the determinants of a matrix generated by the $k^{\text {th }}$ and $l^{\text {th }}$ column of the matrix

$$
v=\left(\begin{array}{cccc}
k & l & m & n \\
k_{1} & l_{1} & m_{1} & n_{1}
\end{array}\right) .
$$

Now it is a matter of long and tedious calculation to prove that the map $\phi:\left(x_{1}, x_{2}, x_{3}\right) \mapsto$ $\left(X_{1}, X_{2}, x_{3}\right)$, where $X_{1}, X_{2}$ are given by (2.3) coincides with the map $R_{12}$ of (2.1). Similarly we can work on $R_{13}$ and $R_{23}$.

3. Since the map $R_{12}:\left(x_{1}, x_{2}, x_{3}\right) \mapsto\left(X_{1}, X_{2}, x_{3}\right)$ satisfies $(2.2)$, the proof of involutivity follows.

4. It is enough to prove that the map $R_{12}$ anti-preserves the measure with density $m_{1}=n^{1} d^{2}$, i.e., the Jacobian determinant

$$
\frac{\partial\left(X_{1}, X_{2}\right)}{\partial\left(x_{1}, x_{2}\right)}:=\left|\begin{array}{ll}
\frac{\partial X_{1}}{\partial x_{1}} & \frac{\partial X_{1}}{\partial x_{2}} \\
\frac{\partial X_{2}}{\partial x_{1}} & \frac{\partial X_{2}}{\partial x_{2}}
\end{array}\right|
$$

equals

$$
\frac{\partial\left(X_{1}, X_{2}\right)}{\partial\left(x_{1}, x_{2}\right)}=-\frac{n^{1}\left(X_{1}, X_{2}, x_{3}\right) d^{2}\left(X_{1}, X_{2}, x_{3}\right)}{n^{1}\left(x_{1}, x_{2}, x_{3}\right) d^{2}\left(x_{1}, x_{2}, x_{3}\right)} .
$$

Since the functions $H_{i}=n^{i} / d^{i}, i=1,2$ are invariant under the action of the map $R_{12}$, it holds

$$
\begin{aligned}
& n^{1}\left(X_{1}, X_{2}, x_{3}\right)=\kappa\left(x_{1}, x_{2}, x_{3}\right) n^{1}\left(x_{1}, x_{2}, x_{3}\right), \\
& d^{1}\left(X_{1}, X_{2}, x_{3}\right)=\kappa\left(x_{1}, x_{2}, x_{3}\right) d^{1}\left(x_{1}, x_{2}, x_{3}\right), \\
& n^{2}\left(X_{1}, X_{2}, x_{3}\right)=\lambda\left(x_{1}, x_{2}, x_{3}\right) n^{2}\left(x_{1}, x_{2}, x_{3}\right), \\
& d^{2}\left(X_{1}, X_{2}, x_{3}\right)=\lambda\left(x_{1}, x_{2}, x_{3}\right) d^{2}\left(x_{1}, x_{2}, x_{3}\right),
\end{aligned}
$$

where $\kappa, \lambda$ are rational functions of $x_{1}, x_{2}, x_{3}$. So,

$$
\frac{n^{1}\left(X_{1}, X_{2}, x_{3}\right) d^{2}\left(X_{1}, X_{2}, x_{3}\right)}{n^{1}\left(x_{1}, x_{2}, x_{3}\right) d^{2}\left(x_{1}, x_{2}, x_{3}\right)}=\kappa\left(x_{1}, x_{2}, x_{3}\right) \lambda\left(x_{1}, x_{2}, x_{3}\right) .
$$

We differentiate equations (2.4) with respect to $x_{1}$ and we eliminate $\frac{\partial \kappa\left(x_{1}, x_{2}, x_{3}\right)}{\partial x_{1}}$ and $\frac{\partial \lambda\left(x_{1}, x_{2}, x_{3}\right)}{\partial x_{1}}$ to obtain

$$
\begin{aligned}
& \frac{1}{n^{1}}\left(\frac{\partial \tilde{n}^{1}}{\partial x_{1}}-\kappa \frac{\partial n^{1}}{\partial x_{1}}\right)=\frac{1}{d^{1}}\left(\frac{\partial \tilde{d}^{1}}{\partial x_{1}}-\kappa \frac{\partial d^{1}}{\partial x_{1}}\right), \\
& \frac{1}{n^{2}}\left(\frac{\partial \tilde{n}^{2}}{\partial x_{1}}-\lambda \frac{\partial n^{2}}{\partial x_{1}}\right)=\frac{1}{d^{2}}\left(\frac{\partial \tilde{d}^{2}}{\partial x_{1}}-\lambda \frac{\partial d^{2}}{\partial x_{1}}\right),
\end{aligned}
$$

here we have suppressed the dependency of $\kappa, \lambda, n^{i}, d^{i}$ on $x_{1}, x_{2}, x_{3}$. By $\tilde{n}^{i}$ we denote $\tilde{n}^{i}:=$ $n^{i}\left(X_{1}, X_{2}, x_{3}\right), i=1,2$, and similarly for $\tilde{d}^{i}$. Also if we differentiate the equations (2.4) with respect to $x_{2}$ and eliminate $\frac{\partial \kappa}{\partial x_{2}}$ and $\frac{\partial \lambda}{\partial x_{2}}$ we obtain

$$
\frac{1}{n^{1}}\left(\frac{\partial \tilde{n}^{1}}{\partial x_{2}}-\kappa \frac{\partial n^{1}}{\partial x_{2}}\right)=\frac{1}{d^{1}}\left(\frac{\partial \tilde{d}^{1}}{\partial x_{2}}-\kappa \frac{\partial d^{1}}{\partial x_{2}}\right),
$$




$$
\frac{1}{n^{2}}\left(\frac{\partial \tilde{n}^{2}}{\partial x_{2}}-\lambda \frac{\partial n^{2}}{\partial x_{2}}\right)=\frac{1}{d^{2}}\left(\frac{\partial \tilde{d}^{2}}{\partial x_{2}}-\lambda \frac{\partial d^{2}}{\partial x_{2}}\right) .
$$

Due to the form of $n^{i}, d^{i}, i=1,2$, equations (2.6), (2.7) are linear in $\frac{\partial X_{1}}{\partial x_{i}}, \frac{\partial X_{2}}{\partial x_{i}}, i=1,2$. Hence we obtain $\frac{\partial X_{1}}{\partial x_{i}}, \frac{\partial X_{2}}{\partial x_{i}}, i=1,2$, in terms of $X_{1}, X_{2}, x_{1}, x_{2}, x_{3}, \kappa, \lambda$ and by using (2.3), the Jacobian determinant reads $\frac{\partial\left(X_{1}, X_{2}\right)}{\partial\left(x_{1}, x_{2}\right)}=-\kappa \lambda$. Using (2.5) we have

$$
\frac{\partial\left(X_{1}, X_{2}\right)}{\partial\left(x_{1}, x_{2}\right)}=-\kappa \lambda=-\frac{\tilde{n}^{1} \tilde{d}^{2}}{n^{1} d^{2}}
$$

that completes the proof. Note that the same holds true for the remaining maps $R_{i j}$.

5. In [3] Adler presented a computational proof based on the fact that the maps $R_{i j}$ map points that lie on the invariant curve

$$
n^{1}\left(x_{1}, x_{2}, x_{3}\right)-C_{1} d^{1}\left(x_{1}, x_{2}, x_{3}\right)=0, \quad n^{2}\left(x_{1}, x_{2}, x_{3}\right)-C_{2} d^{2}\left(x_{1}, x_{2}, x_{3}\right)=0,
$$

that is the intersection of two surfaces of the form $A: N\left(x_{1}, x_{2}, x_{3}\right)=0$, where $N$ is polynomial with degree at most one on each variable $x_{1}, x_{2}$ and $x_{3}$. In [3], it was proven that any surface of the form $A$ that passes through the following five points

$$
\left(\hat{x}_{1}, \tilde{x}_{2}, \hat{\tilde{x}}_{3}\right) \stackrel{R_{13}}{\longleftarrow}\left(x_{1}, \tilde{x}_{2}, \tilde{x}_{3}\right) \stackrel{R_{23}}{\longleftarrow}\left(x_{1}, x_{2}, x_{3}\right) \stackrel{R_{12}}{\longrightarrow}\left(\bar{x}_{1}, \bar{x}_{2}, x_{3}\right) \stackrel{R_{13}}{\longrightarrow}\left(\hat{\bar{x}}_{1}, \bar{x}_{2}, \hat{x}_{3}\right)
$$

passes as well through the point $\left(\hat{\bar{x}}_{1}, Y, \tilde{\hat{x}}_{3}\right)$, that is the point of intersection of the straight line $L:(X, Z)=\left(\hat{\bar{x}}_{1}, \tilde{\hat{x}}_{3}\right)$ and the surface $A$, i.e., $L \cap A=\left(\hat{\bar{x}}_{1}, Y, \tilde{\hat{x}}_{3}\right)$. Since the invariant curve $(2.8)$ is the intersection of two surfaces of the form $A$, it also passes through the point $\left(\hat{\bar{x}}_{1}, Y, \tilde{\hat{x}}_{3}\right)$ and there is $\tilde{\bar{x}}_{2}=Y$. So the values of $\tilde{\bar{x}}_{2}$ obtained in two different ways coincide and this is sufficient for the proof.

Alternatively, one can show by direct computation that the maps $T_{1}=R_{13} R_{12}$ and $T_{2}=$ $R_{12} R_{23}$, commute, i.e., $T_{1} T_{2}=T_{2} T_{1}$. So there is

$$
R_{13} R_{23}=R_{12} R_{23} R_{13} R_{12}
$$

and due to the fact that the maps $R_{i j}$ are involutions, $R_{i j}^{2}=\mathrm{id}$, from the equation above we obtain

$$
R_{12} R_{13} R_{23}=R_{23} R_{13} R_{12} .
$$

Among all the maps that can be constructed by the involutions $R_{i j}$, the following maps

$$
T_{1}=R_{13} R_{12}, \quad T_{2}=R_{12} R_{23}, \quad T_{3}=R_{23} R_{13}
$$

are of special interest since they are not periodic and moreover they satisfy [3]

$$
T_{1} T_{2} T_{3}=\mathrm{id}, \quad T_{i} T_{j}=T_{j} T_{i}, \quad i, j \in\{1,2,3\} .
$$

Proposition 2.2. For the maps $T_{i}, i=1,2,3$ it holds:

1) they preserve the functions $H_{1}, H_{2}$,

2) they are measure-preserving with densities $m_{1}, m_{2}$,

3) they preserve the following degenerate Poisson tensors,

$$
\Omega_{i}^{j}=m_{j}\left(\frac{\partial H_{i}}{\partial x_{3}} \frac{\partial}{\partial x_{1}} \wedge \frac{\partial}{\partial x_{2}}-\frac{\partial H_{i}}{\partial x_{2}} \frac{\partial}{\partial x_{1}} \wedge \frac{\partial}{\partial x_{3}}+\frac{\partial H_{i}}{\partial x_{1}} \frac{\partial}{\partial x_{2}} \wedge \frac{\partial}{\partial x_{3}}\right), \quad i, j \in\{1,2\},
$$

where it holds

$$
0=\Omega_{1}^{j} \nabla H_{1}, \quad \Omega_{1}^{j} \nabla H_{2}=-\Omega_{2}^{j} \nabla H_{1}, \quad \Omega_{2}^{j} \nabla H_{2}=0, \quad j=1,2,
$$


4) they are Liouville integrable maps.

Proof. The statements (1), (2) follows from Proposition 2.1. To prove the statement (3), (4), first note that since the maps $T_{i}$ are measure preserving, they preserve the following polyvector fields

$$
V^{j}=m_{j} \frac{\partial}{\partial x_{1}} \wedge \frac{\partial}{\partial x_{2}} \wedge \frac{\partial}{\partial x_{3}} .
$$

Hence, the contractions $\left.V^{j}\right\rfloor d H_{i}, i, j \in\{1,2\}$ (see [29, 55]) are degenerate Poisson tensors. Namely,

$$
\begin{aligned}
\Omega_{i}^{j} & \left.=\left(m_{j} \frac{\partial}{\partial x_{1}} \wedge \frac{\partial}{\partial x_{2}} \wedge \frac{\partial}{\partial x_{3}}\right)\right\rfloor d H_{i} \\
& =m_{j}\left(\frac{\partial H_{i}}{\partial x_{3}} \frac{\partial}{\partial x_{1}} \wedge \frac{\partial}{\partial x_{2}}-\frac{\partial H_{i}}{\partial x_{2}} \frac{\partial}{\partial x_{1}} \wedge \frac{\partial}{\partial x_{3}}+\frac{\partial H_{i}}{\partial x_{1}} \frac{\partial}{\partial x_{2}} \wedge \frac{\partial}{\partial x_{3}}\right),
\end{aligned}
$$

where $i, j \in\{1,2\}$.

(5) The maps $T_{i}$ preserve the Poisson tensors $\Omega_{i}^{j}$ and the 2 invariants $H_{1}, H_{2}$, so they are Liouville integrable maps [14, 52, 68].

Note that on the level surfaces $H_{2}\left(x_{1}, x_{2}, x_{3}\right)=c$, maps $T_{1}, T_{2}, T_{3}$ reduce to pair-wise commuting maps on the plane which preserve the function $\hat{H}_{1}\left(x_{1}, x_{2} ; c\right)$. One of these reduced maps is the associated with the invariant $\hat{H}_{1}\left(x_{1}, x_{2} ; c\right)$ QRT map. Examples of commuting maps with specific members of the QRT family of maps were also constructed in [30].

The involution $R_{12}$ under the reduction $x_{2}=x_{1}, H_{2}=H_{1}=H$, so $H=\frac{n}{d}=\frac{a x_{1}^{2}+b x_{1}+c}{k x_{1}^{2}+l x_{1}+m}$, reads

$$
R_{12}:\left(x_{1}, x_{3}\right) \mapsto\left(x_{1}-2 \frac{D_{x_{1}} n \cdot d}{\partial_{x_{1}} D_{x_{1}} n \cdot d}, x_{3}\right),
$$

that coincides with the QRT involution $i_{x}$ that preserves the invariant $H$. This formulae for the QRT involution $i_{x}$ was firstly given in [37], where an elegant presentation of the QRT map was considered.

\subsection{A generalisation of the triad family of maps}

Following the same generalisation procedures introduced for the QRT family of maps [15, 29, $35,62,67]$, the triad family of maps can be generalised in similar manners. Here, in order to generalise the triad family of maps, we mimic the generalisation of the QRT family of maps introduced in [67].

Consider the following polynomials

$$
\begin{aligned}
n^{i} & =\sum_{j_{1}, j_{2}, \ldots, j_{k}=0}^{1} \alpha_{j_{1}, j_{2}, \ldots, j_{k}}^{i} x_{1}^{1-j_{1}} x_{2}^{1-j_{2}} \cdots x_{k}^{1-j_{k}}, \\
d^{i} & =\sum_{j_{1}, j_{2}, \ldots, j_{k}=0}^{1} \beta_{j_{1}, j_{2}, \ldots, j_{k}}^{i} x_{1}^{1-j_{1}} x_{2}^{1-j_{2}} \cdots x_{k}^{1-j_{k}}, \quad i=1,2 k \geq 3,
\end{aligned}
$$

where $x_{1}, x_{2}, \ldots, x_{k}$ are considered as variables and $\alpha_{j_{1}, j_{2}, \ldots, j_{k}}^{i}, \beta_{j_{1}, j_{2}, \ldots, j_{k}}^{i}$ as parameters. We consider the $\left(\begin{array}{l}k \\ 2\end{array}\right)$ maps $R_{i j}, i<j, i, j \in\{1,2, \ldots, k\}$. These maps can be build out of the polynomials $n^{i}, d^{i}$ and they read: $R_{i j}:\left(x_{1}, x_{2}, \ldots, x_{k}\right) \mapsto\left(X_{1}, X_{2}, \ldots, X_{k}\right)$, where $X_{l}=x_{l}$ $\forall l \neq i, j$ and $X_{i}, X_{j}$ are given by the formulae (2.1), where $n^{i}, d^{i}, i=1,2$ are given by (2.9).

Proposition 2.1 is straight forward extended to the $k$-variables case. 
Proposition 2.3. The following holds:

1. Mappings $R_{i j}$ depend on $4 \cdot 2^{k}$ parameters $\alpha_{j_{1}, j_{2}, \ldots, j_{k}}^{i}, \beta_{j_{1}, j_{2}, \ldots, j_{k}}^{i}, i=1,2, j_{1}, j_{2}, \ldots, j_{k} \in$ $\{0,1\}$. Only $4 \cdot 2^{k}-3 k-8$ of them are essential.

2. The functions $H_{1}=n^{1} / d^{1}, H_{2}=n^{2} / d^{2}$ are invariant under the action of $R_{i j}$, i.e., $H_{l} \circ$ $R_{i j}=H_{l}, l=1,2$.

3. Mappings $R_{i j}$ are involutions, i.e., $R_{i j}^{2}=\mathrm{id}$.

4. Mappings $R_{i j}$ are anti-measure preserving with densities $m_{1}=n^{1} d^{2}, m_{2}=n^{2} d^{1}$.

5. Mappings $R_{m n}, m<n, m, n \in\{1,2, \ldots, k\}$ satisfy the relations $R_{i j} R_{i l} R_{j l}=R_{j l} R_{i l} R_{i j}$.

Proof. 1. The invariants $H_{1}, H_{2}$ depend on $k \geq 3$ variables and they include $4 \cdot 2^{k}$ parameters. Acting with a different Möbius transformation to each of the variables, $3 k$ parameters can be removed. A Möbius transformation of an invariant remains an invariant, since we have 2 invariants, 6 more parameters can be removed. Finally, since any multiple of an invariant remains an invariant, 2 more parameters can be removed. That leaves us with $4 \cdot 2^{k}-3 k-6-2=$ $4 \cdot 2^{k}-3 k-8$ essential parameters for the invariants $H_{1}, H_{2}$ and hence for the maps $R_{i j}$.

The proof of the remaining statements of this Proposition follows directly from the fact that for any 3 indices $p<q<r \in\{1,2, \ldots, k\}$, the maps $R_{p q}, R_{p r}$ and $R_{q r}$, coincide with the maps $R_{12}, R_{13}$ and $R_{23}$ respectively of Proposition 2.1.

We take a stand here to comment that for $k=3$ the construction above coincides with the Adler's triad family of maps hence we have Liouville integrability. For $k>3$ we have a generalisation of the latter and since always we will have maps in $k$ variables with 2 invariants, Liouville integrability is not expected for generic choice of the parameters $\alpha_{j_{1}, j_{2}, \ldots, j_{k}}^{i}, \beta_{j_{1}, j_{2}, \ldots, j_{k}}^{i}$. For a specific but quite general choice of the parameters though, one can associate a Lax pair to these maps and recover the additional integrals which are required for the Liouville integrability to emerge.

We also have to note that the case $k=4$ was firstly introduced in [43]. Although for $k=4$ we have mappings in 4 variables with 2 invariants, Liouville integrability is not apparent unless we specify the parameters. A specific choice of the parameters which leads to integrability is presented to the following example.

Example 2.4 (the Adler-Yamilov map [7]). Consider the following special form of the functions $n^{i}, d^{i}$

$$
d^{1}=d^{2}=1, \quad n^{1}=x_{1} x_{2}+x_{3} x_{4}, \quad n^{2}=x_{1} x_{2} x_{3} x_{4}+x_{1} x_{4}+x_{2} x_{3}+a x_{1} x_{2}+b x_{3} x_{4} .
$$

Then the functions $H_{i}=n^{i} / d^{i}, i=1,2$ are preserved by construction by the maps $R_{i j}$ as well as by the following elementary involutions

$$
i:\left(x_{1}, x_{2}, x_{3}, x_{4}\right) \mapsto\left(x_{2}, x_{1}, x_{4}, x_{3}\right), \quad \phi:\left(x_{1}, x_{2}, x_{3}, x_{4}\right) \mapsto\left(x_{1} x_{2} / x_{3}, x_{3}, x_{2}, x_{3} x_{4} / x_{2}\right) .
$$

The Adler-Yamilov map $(\xi)$ is considered by the following composition

$$
\xi:=R_{14} \phi i: \quad\left(x_{1}, x_{2}, x_{3}, x_{4}\right) \mapsto\left(x_{3}-\frac{(a-b) x_{1}}{1+x_{1} x_{4}}, x_{4}, x_{1}, x_{2}+\frac{(a-b) x_{4}}{1+x_{1} x_{4}}\right) .
$$

The Adler-Yamilov map is Liouville integrable since it preserves, and the invariants $H_{1}, H_{2}$ are in involution with respect to the canonical Poisson bracket. For further discussions on the Adler-Yamilov map see [30, 48]. 


\section{Invariants in separated variables and Yang-Baxter maps}

Mappings $R_{m n}, m<n \in\{1,2, \ldots, k\}$, presented in Section 2.1, satisfy the identities $R_{i j} R_{i l} R_{j l}=$ $R_{j l} R_{i l} R_{i j}$, nevertheless as they stand they are not Yang-Baxter. Take for example the map $R_{12}:\left(x_{1}, x_{2}, x_{3}, \ldots, x_{k}\right) \mapsto\left(X_{1}, X_{2}, x_{3}, \ldots, x_{k}\right)$. The formulae for $X_{1}$ is fraction linear in $x_{1}$ with coefficients that depend on all the remaining variables and $X_{2}$ is fraction linear in $x_{2}$ with coefficients that depend on all the remaining variables. In order for $R_{12}$ to be a Yang-Baxter map the coefficients of $x_{1}$ in the formulae of $X_{1}$ should depend only on $x_{2}$ and the coefficients of $x_{2}$ in the formulae of $X_{2}$ should depend only on $x_{1}$. This "separability" requirement can be easily achieved by requiring separability of variables on the level of the invariants of the map $R_{12}$. We have two invariants $H_{1}=n^{1} / d^{1}, H_{2}=n^{2} / d^{2}$, so we can have three different kinds of separability. (I) Both $H_{1}$ and $H_{2}$ to be multiplicative separable on the variables $x_{1}$ and $x_{2}$. (II) $H_{1}$ to be multiplicative and $H_{2}$ to be additive separable and finally (III) both $H_{1}$ and $H_{2}$ to be additive separable on the variables $x_{1}$ and $x_{2}$. In what follows we explicitly present these three different kinds of separability in all variables of the invariants $H_{1}$ and $H_{2}$.

(I) Multiplicative/multiplicative separability of variables:

$$
H_{1}=\prod_{i=1}^{k} \frac{a_{i}-b_{i} x_{i}}{c_{i}-d_{i} x_{i}}, \quad H_{2}=\prod_{i=1}^{k} \frac{A_{i}-B_{i} x_{i}}{C_{i}-D_{i} x_{i}} .
$$

(II) Multiplicative/additive separability of variables:

$$
H_{1}=\prod_{i=1}^{k} \frac{a_{i}-b_{i} x_{i}}{c_{i}-d_{i} x_{i}}, \quad H_{2}=\sum_{i=1}^{k} \frac{A_{i}-B_{i} x_{i}}{C_{i}-D_{i} x_{i}} .
$$

(III) Additive/additive separability of variables:

$$
H_{1}=\sum_{i=1}^{k} \frac{a_{i}-b_{i} x_{i}}{c_{i}-d_{i} x_{i}}, \quad H_{2}=\sum_{i=1}^{k} \frac{A_{i}-B_{i} x_{i}}{C_{i}-D_{i} x_{i}} .
$$

In the formulas above, $a_{i}, b_{i}, c_{i}, d_{i}, A_{i}, B_{i}, C_{i}, D_{i}, i=1, \ldots, k$ are parameters, $8 k$ in total. In all three cases above, the number of essential parameters is $3 k-6$. This argument can be proven by the following reasoning. Since the invariants $H_{1}, H_{2}$ depends on $k$ variables, by a Möbius transformation on each of the $k$ variables $3 k$ parameters can be removed. Also any Möbius transformation of an invariant remains an invariant so since we have two invariants $2 \times 3$ more parameters can be removed. Finally, for each one of the $2 k$ functions $\frac{a_{i}-b_{i} x_{i}}{c_{i}-d_{i} x_{i}}, \frac{A_{i}-B_{i} x_{i}}{C_{i}-D_{i} x_{i}}, i=$ $1, \ldots, k$, one non-zero parameter can be absorbed simply by dividing with it (and reparametrise), so $2 k$ more parameters can be removed. In total we have $8 k-3 k-2 \times 3-2 k=3 k-6$ essential parameters.

\subsection{Multiplicative/multiplicative separability of variables}

Let us first introduce some definitions.

Definition 3.1. The maps $R, \tilde{R}: \mathbb{C P}^{1} \times \mathbb{C P}^{1} \mapsto \mathbb{C P}^{1} \times \mathbb{C P}^{1}$ are (Möb) $)^{2}$ equivalent if there exists bijections $\phi, \psi: \mathbb{C P}^{1} \mapsto \mathbb{C P}^{1}$ such that the following conjugation relation holds

$$
\tilde{R}=\phi^{-1} \times \psi^{-1} R \phi \times \psi
$$


Definition 3.2. The map $R$ : $\mathbb{C P}^{1} \times \mathbb{C P}^{1} \ni(u, v) \mapsto(U, V) \in \mathbb{C P}^{1} \times \mathbb{C P}^{1}$, where

$$
U=\frac{a_{1}+a_{2} u}{a_{3}+a_{4} u}, \quad V=\frac{b_{1}+b_{2} v}{b_{3}+b_{4} v},
$$

with $a_{i}, b_{i}, i=1, \ldots, 4$ known polynomials of $v$ and $u$ respectively, will be said to be of subclass $[\gamma: \delta]$, if the highest degree that appears in the polynomials $a_{i}$ is $\gamma$ and the higher degree that appears in the polynomials $b_{i}$ is $\delta$.

Clearly, maps that belong to different subclasses are not (Möb) ${ }^{2}$ equivalent.

Proposition 3.3. Consider the multiplicative/multiplicative separability of variables of the invariants $H_{1}$ and $H_{2}$ (see (3.1)). Consider also the following sets of parameters

$$
\mathbf{p}_{i j}:=\mathbf{p}_{i} \cup \mathbf{p}_{j} \quad \text { where } \quad \mathbf{p}_{i}:=\left\{a_{i}, b_{i}, c_{i}, d_{i}, A_{i}, B_{i}, C_{i}, D_{i}\right\}, \quad i<j \in\{1,2, \ldots, k\}
$$

and the functions

$$
f_{i}:=\frac{a_{i}-b_{i} x_{i}}{c_{i}-d_{i} x_{i}}, \quad g_{i}:=\frac{A_{i}-B_{i} x_{i}}{C_{i}-D_{i} x_{i}}, \quad i=1, \ldots, k .
$$

The following holds:

1. The invariants $H_{1}=\prod_{i=1}^{k} f_{i}, H_{2}=\prod_{i=1}^{k} g_{i}$ depend on $8 k$ parameters. Only $3 k-6$ of them are essential.

2. Mappings $R_{i j}$ explicitly read

$$
R_{i j}:\left(x_{1}, x_{2}, \ldots, x_{k}\right) \mapsto\left(X_{1}, X_{2}, \ldots, X_{k}\right),
$$

where $X_{l}=x_{l} \forall l \neq i, j$ and $X_{i}, X_{j}$ are given by the formulae

$$
\begin{aligned}
& X_{i}=x_{i}-2 \frac{\left|\begin{array}{ll}
f_{i}^{\prime} f_{j} & f_{i} f_{j}^{\prime} \\
g_{i}^{\prime} g_{j} & g_{i} g_{j}^{\prime}
\end{array}\right|}{g_{i}^{\prime} g_{j}\left(\frac{f_{i}^{\prime}}{f_{j}^{\prime}}\left|\begin{array}{cc}
f_{j} & f_{j}^{\prime} \\
f_{j}^{\prime} & f_{j}^{\prime \prime}
\end{array}\right|+\frac{f_{j}^{\prime}}{f_{i}^{\prime}}\left|\begin{array}{cc}
f_{i} & f_{i}^{\prime} \\
f_{i}^{\prime} & f_{i}^{\prime \prime}
\end{array}\right|\right)-f_{i}^{\prime} f_{j}\left(\frac{g_{i}^{\prime}}{g_{j}^{\prime}}\left|\begin{array}{ll}
g_{j} & g_{j}^{\prime} \\
g_{j}^{\prime} & g_{j}^{\prime \prime}
\end{array}\right|+\frac{g_{j}^{\prime}}{g_{i}^{\prime}}\left|\begin{array}{ll}
g_{i} & g_{i}^{\prime} \\
g_{i}^{\prime} & g_{i}^{\prime \prime}
\end{array}\right|\right)},
\end{aligned}
$$

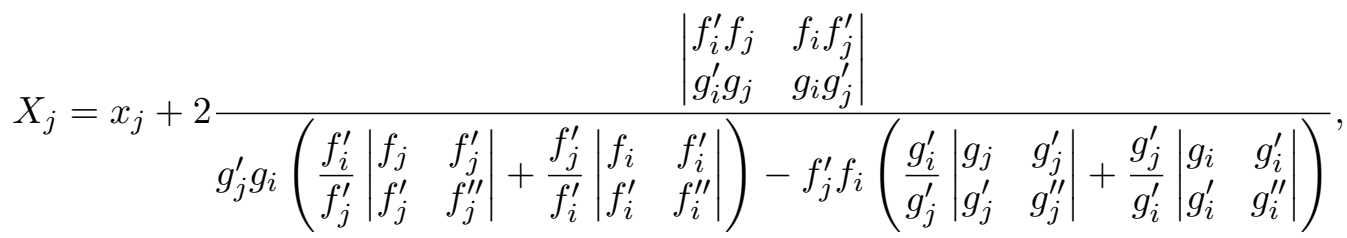

where $f_{l}^{\prime} \equiv \frac{\partial f_{l}}{\partial x_{l}}, g_{l}^{\prime} \equiv \frac{\partial g_{l}}{\partial x_{l}}, g_{l}^{\prime \prime} \equiv \frac{\partial^{2} g_{l}}{\partial x_{l}^{2}}$, etc. Note that in the expressions of $X_{i}, X_{j}$ appears only the coordinates $x_{i}, x_{j}$ and the parameters $\mathbf{p}_{i j}$. From further on we denote the maps $R_{i j}$ as $R_{i j}^{\mathbf{p}_{i j}}$, in order to stress this separability feature.

3. Mappings $R_{i j}^{\mathbf{p}_{i j}}$ are anti-measure preserving with densities $m_{1}=n^{1} d^{2}, m_{2}=n^{2} d^{1}$, where $n^{i}, d^{i}$ the numerators and the denominators respectively, of the invariants $H_{i}, i=1,2$.

4. Mappings $R_{i j}^{\mathbf{p}_{i j}}$ satisfy the Yang-Baxter identity

$$
R_{i j}^{\mathbf{p}_{i j}} R_{i k}^{\mathbf{p}_{i k}} R_{j k}^{\mathbf{p}_{j k}}=R_{j k}^{\mathbf{p}_{j k}} R_{i j}^{\mathbf{p}_{i j}} R_{i j}^{\mathbf{p}_{i j}}
$$


5. Mappings $R_{i j}^{\mathbf{p}_{i j}}$ are involutions with the sets of singularities

$$
\Sigma_{i j}=\left\{P_{i j}^{1}, P_{i j}^{2}, P_{i j}^{3}, P_{i j}^{4}\right\}=\left\{\left(\frac{a_{i}}{b_{i}}, \frac{c_{j}}{d_{j}}\right),\left(\frac{c_{i}}{d_{i}}, \frac{a_{j}}{b_{j}}\right),\left(\frac{A_{i}}{B_{i}}, \frac{C_{j}}{D_{j}}\right),\left(\frac{C_{i}}{D_{i}}, \frac{A_{j}}{B_{j}}\right)\right\},
$$

and the sets of fixed points

$$
\Phi_{i j}=\left\{Q_{i j}^{1}, Q_{i j}^{2}, Q_{i j}^{3}, Q_{i j}^{4}\right\}=\left\{\left(\frac{a_{i}}{b_{i}}, \frac{a_{j}}{b_{j}}\right),\left(\frac{c_{i}}{d_{i}}, \frac{c_{j}}{d_{j}}\right),\left(\frac{A_{i}}{B_{i}}, \frac{A_{j}}{B_{j}}\right),\left(\frac{C_{i}}{D_{i}}, \frac{C_{j}}{D_{j}}\right)\right\},
$$

where in the formulae for $P_{i j}^{m}$ and $Q_{i j}^{m}, m=1, \ldots, 4$, we have suppressed the dependency on the remaining variables. For example, with $P_{i j}^{1}=\left(\frac{a_{i}}{b_{i}}, \frac{c_{j}}{d_{j}}\right)$ we denote $\left(x_{1}, \ldots, x_{i-1}, \frac{a_{i}}{b_{i}}, x_{i+1}\right.$, $\left.\ldots, x_{j-1}, \frac{c_{j}}{d_{j}}, x_{j+1}, \ldots, x_{k}\right)$ and similarly for the remaining $P_{i j}^{m}$ and $Q_{i j}^{m}$.

6. Each one of the maps $R_{i j}^{\mathbf{p}_{i j}}$ is (Möb) ${ }^{2}$ equivalent to the $H_{\mathrm{I}}$ Yang-Baxter map.

Proof. (1) See at the end of the previous subsection.

(2) Mappings (2.1) written in terms of the functions $f_{i}, g_{i}$ get exactly the desired form.

(3) See Proposition 2.1.

(4) See Proposition 2.1.

(5) Because mappings $R_{i j}^{\mathbf{p}_{i j}}$, for generic parameter sets $\mathbf{p}_{i j}$, belong to the $[2: 2]$ subclass, we expect at most 8 singular points, 4 singular points from the first fraction of the map and 4 from the second. By direct calculation we show that the singular points of the first and the second fraction of $R_{i j}^{\mathbf{p}_{i j}}$ coincide. Moreover, $P_{i j}^{m}, m=1, \ldots, 4$ are the singular points of the maps $R_{i j}^{\mathbf{p}_{i j}}$, i.e.,

$$
R_{i j}^{\mathbf{p}_{i j}}: P_{i j}^{m} \mapsto\left(x_{1}, \ldots, x_{i-1}, \frac{0}{0}, x_{i+1}, \ldots, x_{j-1}, \frac{0}{0}, x_{j+1}, \ldots, x_{k}\right) .
$$

Note that the values of the invariants $H_{i}$ at the singular points $P_{i j}^{m}$ are undetermined, i.e., $H_{1}\left(P_{i j}^{m}\right)=\frac{0}{0}, m=1,2, H_{2}\left(P_{i j}^{m}\right)=\frac{0}{0}, m=3,4$. For the fixed points $Q_{i j}^{m}, m=1, \ldots, 4$ it holds $R_{i j}^{\mathbf{p}_{i j}}: Q_{i j}^{m} \mapsto Q_{i j}^{m}$. Note also that $H_{1}\left(Q_{i j}^{1}\right)=0, H_{1}\left(Q_{i j}^{2}\right)=\infty, H_{2}\left(Q_{i j}^{3}\right)=0, H_{2}\left(Q_{i j}^{4}\right)=\infty$.

(6) Introducing the new variables $y_{i}, y_{j}, i \neq j=1, \ldots, k$ though

$$
\begin{aligned}
& \mathrm{CR}\left[x_{i}, a_{i} / b_{i}, c_{i} / d_{i}, A_{i} / B_{i}\right]=\mathrm{CR}\left[y_{i}, 0,1, \infty\right], \\
& \mathrm{CR}\left[x_{j}, c_{j} / d_{j}, a_{j} / b_{j}, C_{j} / D_{j}\right]=\operatorname{CR}\left[y_{j}, \infty, 1,0\right],
\end{aligned}
$$

after a re-parametrization mappings $R_{i j}$ gets exactly the form of the $H_{\mathrm{I}}$ map. Here, with $\mathrm{CR}[a, b, c, d]$ we denote the cross-ratio of 4 points, namely

$$
\mathrm{CR}[a, b, c, d]:=\frac{(a-c)(b-d)}{(a-d)(b-c)} .
$$

Each one of the maps $R_{i j}$ has a set of singularities which consists of 4 distinct points. With appropriate limits we are allowed to merge some of the singularities and obtain Yang-Baxter maps which are not (Möb) ${ }^{2}$ equivalent with the original one.

By setting $C_{i}=\epsilon A_{i}, D_{i}=\epsilon B_{i}, A_{j}=\epsilon C_{j}, B_{j}=\epsilon D_{j}$ and letting $\epsilon \rightarrow 0$ the singular points $P_{i j}^{4}$ and $P_{i j}^{3}$ merge. The resulting maps, under a re-parametrization, coincide with the ones obtained in the multiplicative/additive case (see Section 3.2), hence are (Möb) ${ }^{2}$ equivalent with the $H_{\mathrm{II}}$ Yang-Baxter map. The same result can be obtained by merging $P_{i j}^{2}$ and $P_{i j}^{1}$. Note that merging $P_{i j}^{4}$ with $P_{i j}^{2}$ or $P_{i j}^{4}$ with $P_{i j}^{1}$ is not of interest since the resulting maps are trivial.

By further setting $c_{i}=\epsilon a_{i}, d_{i}=\epsilon b_{i}, a_{j}=\epsilon c_{j}, b_{j}=\epsilon d_{j}$ and letting $\epsilon \rightarrow 0$ the singular points $P_{i j}^{2}$ and $P_{i j}^{1}$ merge as well. The resulting maps, under a re-parametrization, coincide with the ones obtained in the additive/additive case (see Section 3.3), hence are (Möb) ${ }^{2}$ equivalent with the $H_{\mathrm{III}}^{A}$ Yang-Baxter map. Any further merging of singularities leads to trivial maps. 
Remark 3.4. An interesting observation is that if we impose that the fixed points $Q_{i j}^{4}$ of the maps $R_{i j}$ coincide with the singular points $P_{i j}^{2}$ or the fixed points $Q_{i j}^{4}$ coincide with $P_{i j}^{1}$, we obtain maps which belong to the $[1: 1]$ subclass of maps. The same is true if we demand that the fixed points $Q_{i j}^{1}$ coincide with the singular points $P_{i j}^{3}$ or if the fixed points $Q_{i j}^{1}$ coincide with the singular points $P_{i j}^{4}$,

Remark 3.5. For generic sets of parameters $\mathbf{p}_{i j}$, each one of the $\left(\begin{array}{l}k \\ 2\end{array}\right)$ maps $R_{i j}^{\mathbf{p}_{i j}}$, is (Möb) ${ }^{2}$ equivalent to the $H_{\mathrm{I}}$ Yang-Baxter map. For degenerate choices of the sets $\mathbf{p}_{i j}$, this is no longer the case. Hence, in that respect, mappings $R_{i j}^{\mathbf{p}_{i j}}$ are more general than the $H_{\mathrm{I}}$ map since they include degenerate cases as well. In the same respect $Q_{\mathrm{V}}$ [72], the rational version of the discrete Krichever-Novikov equation $Q_{4}$ [2], is more general.

Example 3.6 $(k=3)$. For $k=3$, the invariants $H_{1}=f_{1} f_{2} f_{3}, H_{2}=g_{1} g_{2} g_{3}$ are functions of 3 variables with 24 parameters, 3 of them are essential. Without loss of generality, after removing the redundancy of the parameters, the invariants $H_{1}, H_{2}$ can be cast into the form

$$
H_{1}=x_{1} x_{2} x_{3}, \quad H_{2}=\frac{x_{1}-p_{1}}{x_{1}-1} \frac{x_{2}-p_{2}}{x_{2}-1} \frac{x_{3}-p_{3}}{x_{3}-1} .
$$

Then each of the mappings $R_{i j}, i \neq j \in\{1,2,3\}$ is exactly the $H_{\mathrm{I}}$ Yang-Baxter map. The $H_{\mathrm{I}}$ Yang-Baxter map explicitly reads $H_{\mathrm{I}}:(u, v) \mapsto(U, V)$ where

$$
U=v Q, \quad V=u Q^{-1}, \quad Q=\frac{(\alpha-1) u v+(\beta-\alpha) u+\alpha(1-\beta)}{(\beta-1) u v+(\alpha-\beta) v+\beta(1-\alpha)} .
$$

By the identifications $u \equiv x_{i}, u \equiv x_{j}, \alpha \equiv p_{i}$ and $\beta \equiv p_{j}$, from (3.4) we recover the maps $R_{i j}$.

The maps $\phi_{i}:\left(x_{1}, x_{2}, x_{3}\right) \mapsto\left(X_{1}, X_{2}, X_{3}\right)$ where $X_{l}=x_{l} \forall l \neq i$ and $X_{i}=\frac{p_{i}}{x_{i}}, i=1,2,3$ and the maps $\psi_{i}:\left(x_{1}, x_{2}, x_{3}\right) \mapsto\left(X_{1}, X_{2}, X_{3}\right)$ where $X_{l}=x_{l} \forall l \neq i$ and $X_{i}=\frac{x_{i}-p_{i}}{x_{i}-1}, i=1,2,3$ satisfy

$$
H_{1} \phi_{1} \phi_{2} \phi_{3}=\frac{p_{1} p_{2} p_{3}}{H_{1}}, \quad H_{2} \phi_{1} \phi_{2} \phi_{3}=\frac{p_{1} p_{2} p_{3}}{H_{2}}, \quad H_{1} \psi_{1} \psi_{2} \psi_{3}=H_{2}, \quad H_{2} \psi_{1} \psi_{2} \psi_{3}=H_{1} \text {. }
$$

The maps $\phi_{i}$ and $\psi_{i}$ have a special role in [59] since though them the $H_{\text {I }}$ map was derived out of the $F_{\mathrm{I}}$ Yang-Baxter map. We will discuss more about these maps in the next Section. We just quickly recall that $\phi_{1} R_{12} \phi_{2}$ is exactly the $F_{\mathrm{I}}$ Yang-Baxter map.

Remark 3.7. We have to remark that with loss of generality, mappings $R_{i j}$ can belong on a different subclasses than the $[2: 2]$ subclass of maps that the $H_{\text {I }}$ map belongs to. For example, for

$$
H_{1}=\left(x_{1}-p_{1}\right)\left(x_{2}-p_{2}\right)\left(x_{3}-p_{3}\right), \quad H_{2}=\frac{x_{1}-p_{1}}{x_{1}} \frac{x_{2}}{x_{2}-p_{2}} \frac{x_{3}}{x_{3}-1},
$$

$R_{12}$ is the Hirota's KdV map (see [44]) that belongs on the subclass [1:1] and $R_{13}, R_{23}$ are maps which belong to the subclass $[2: 1]$. Explicitly the maps read

$$
\begin{aligned}
& R_{12}:\left(x_{1}, x_{2}, x_{3}\right) \mapsto\left(\frac{p_{1}\left(p_{2} x_{1}+p_{1} x_{2}-x_{1} x_{2}\right)}{p_{2} x_{1}}, \frac{p_{2}\left(p_{2} x_{1}+p_{1} x_{2}-x_{1} x_{2}\right)}{p_{1} x_{2}}, x_{3}\right), \\
& R_{13} \equiv S_{13}:\left(x_{1}, x_{2}, x_{3}\right) \mapsto\left(\frac{p_{1}\left(-1+x_{3}\right)\left(p_{3} x_{1}+p_{1} x_{3}-x_{1} x_{3}\right)}{-p_{3} x_{1}-p_{1} x_{3}+p_{1} p_{3} x_{3}+x_{1} x_{3}}, x_{2}, \frac{p_{3} x_{1}+p_{1} x_{3}-x_{1} x_{3}}{p_{1} x_{3}}\right), \\
& R_{23} \equiv T_{23}:\left(x_{1}, x_{2}, x_{3}\right) \mapsto\left(x_{1}, \frac{p_{2} x_{3}\left(-p_{2}+p_{3} x_{2}+p_{2} x_{3}-x_{2} x_{3}\right)}{-p_{2} p_{3}+p_{3} x_{2}+p_{2} p_{3} x_{3}-x_{2} x_{3}}, \frac{x_{2}\left(-p_{3}+x_{3}\right)}{p_{2}\left(-1+x_{3}\right)}\right) .
\end{aligned}
$$

The Hirota's KdV map entwines with $S_{13}$ and $T_{23}$, since $R_{12} S_{13} T_{23}=T_{23} S_{13} R_{12}$ holds. 
Example $3.8(k \geq 4)$. For $k=4$ the invariants depend on 32 parameters and only 6 of them are essential. Without loss of generality they can be cast into the form

$$
H_{1}=x_{1} x_{2} x_{3} x_{4}, \quad H_{2}=\frac{x_{1}-p_{1}}{x_{1}-1} \frac{x_{2}-p_{2}}{x_{2}-1} \frac{x_{3}-p_{3}}{x_{3}-1} \frac{\alpha_{4}-\beta_{4} x_{4}}{\beta_{4}-\gamma_{4} x_{4}} .
$$

For $k>4$ the invariants depend on $8 k$ parameters and only $3 k-6$ of them are essential. Without loss of generality they can be cast into the form

$$
H_{1}=\prod_{i=1}^{k} x_{i}, \quad H_{2}=\frac{x_{1}-p_{1}}{x_{1}-1} \frac{x_{2}-p_{2}}{x_{2}-1} \frac{x_{3}-p_{3}}{x_{3}-1} \prod_{i=4}^{k} \frac{\alpha_{i}-\beta_{i} x_{i}}{\beta_{i}-\gamma_{i} x_{i}} .
$$

\subsection{Multiplicative/additive separability of variables}

Proposition 3.9. Consider the multiplicative/additive separability of variables of the invariants $H_{1}$ and $H_{2}$ (see (3.2)). Consider also the following sets of parameters

$$
\mathbf{p}_{i j}:=\mathbf{p}_{i} \cup \mathbf{p}_{j}, \quad \text { where } \quad \mathbf{p}_{i}:=\left\{a_{i}, b_{i}, c_{i}, d_{i}, A_{i}, B_{i}, C_{i}, D_{i}\right\}, \quad i<j \in\{1,2, \ldots, k\}
$$

and the functions

$$
f_{i}:=\frac{a_{i}-b_{i} x_{i}}{c_{i}-d_{i} x_{i}}, \quad g_{i}:=\frac{A_{i}-B_{i} x_{i}}{C_{i}-D_{i} x_{i}}, \quad i=1, \ldots, k .
$$

The following holds:

1. The invariants $H_{1}=\prod_{i=1}^{k} f_{i}, H_{2}=\sum_{i=1}^{k} g_{i}$ depend on $8 k$ parameters. Only $3 k-6$ of them are essential.

2. Mappings $R_{i j}$ explicitly read

$$
R_{i j}:\left(x_{1}, x_{2}, \ldots, x_{k}\right) \mapsto\left(X_{1}, X_{2}, \ldots, X_{k}\right),
$$

where $X_{l}=x_{l} \forall l \neq i, j$ and $X_{i}, X_{j}$ are given by the formulae

$$
X_{i}=x_{i}-2 \frac{\begin{array}{cc}
f_{i} f_{j}^{\prime} & f_{i}^{\prime} f_{j} \\
g_{j}^{\prime} & g_{i}^{\prime}
\end{array} \mid}{\mid \begin{array}{ccc}
f_{i}^{\prime} f_{j} & g_{i}^{\prime} \\
\frac{f_{j}^{\prime}}{f_{i}^{\prime}} f_{i} f_{i}^{\prime \prime}+\frac{f_{i}^{\prime}}{f_{j}^{\prime}} f_{j} f_{j}^{\prime \prime}-2 f_{i}^{\prime} f_{j}^{\prime} & \frac{g_{j}^{\prime}}{g_{i}^{\prime}} g_{i}^{\prime \prime}+\frac{g_{i}^{\prime}}{g_{j}^{\prime}} g_{j}^{\prime \prime} \mid
\end{array}},
$$

where $f_{l}^{\prime} \equiv \frac{\partial f_{l}}{\partial x_{l}}, g_{l}^{\prime} \equiv \frac{\partial g_{l}}{\partial x_{l}}, g_{l}^{\prime \prime} \equiv \frac{\partial^{2} g_{l}}{\partial x_{l}^{2}}$, etc. Note that in the expressions of $X_{i}, X_{j}$ appears only the coordinates $x_{i}, x_{j}$ and the parameters $\mathbf{p}_{i j}$. From further on we denote the maps $R_{i j}$ as $R_{i j}^{\mathbf{p}_{i j}}$, in order to stress this separability feature.

3. Mappings $R_{i j}^{\mathbf{p}_{i j}}$ are anti-measure preserving with densities $m_{1}=n^{1} d^{2}, m_{2}=n^{2} d^{1}$, where $n^{i}, d^{i}$ the numerators and the denominators respectively, of the invariants $H_{i}, i=1,2$. 
4. Mappings $R_{i j}^{\mathbf{p}_{i j}}$ satisfy the Yang-Baxter identity

$$
R_{i j}^{\mathbf{p}_{i j}} R_{i k}^{\mathbf{p}_{i k}} R_{j k}^{\mathbf{p}_{j k}}=R_{j k}^{\mathbf{p}_{j k}} R_{i j}^{\mathbf{p}_{i j}} R_{i j}^{\mathbf{p}_{i j}}
$$

5. Mappings $R_{i j}^{\mathbf{p}_{i j}}$ are involutions with the sets of singularities

$$
\Sigma_{i j}=\left\{P_{i j}^{1}, P_{i j}^{2}, P_{i j}^{3}\right\}=\left\{\left(\frac{a_{i}}{b_{i}}, \frac{c_{j}}{d_{j}}\right),\left(\frac{c_{i}}{d_{i}}, \frac{a_{j}}{b_{j}}\right),\left(\frac{C_{i}}{D_{i}}, \frac{C_{j}}{D_{j}}\right)^{2}\right\},
$$

where the superscript 2 in $P_{i j}^{3}$ denotes that these singular points appears with multiplicity 2. In the formulae for $P_{i j}^{m}, m=1, \ldots, 3$, we have suppressed the dependency on the remaining variables. For example, with $P_{i j}^{1}=\left(\frac{a_{i}}{b_{i}}, \frac{c_{j}}{d_{j}}\right)$ we denote $\left(x_{1}, \ldots, x_{i-1}, \frac{a_{i}}{b_{i}}, x_{i+1}, \ldots, x_{j-1}, \frac{c_{j}}{d_{j}}\right.$, $\left.x_{j+1}, \ldots, x_{k}\right)$ and similarly for the remaining $P_{i j}^{m}$.

6. Each one of the maps $R_{i j}^{\mathbf{p}_{i j}}$ is (Möb) ${ }^{2}$ equivalent to the $H_{\mathrm{II}}$ Yang-Baxter map.

Proof. The proof follows similarly to the proof of Proposition 3.3.

Example $3.10(k \geq 3)$. For $k=3$, the invariants $H_{1}=f_{1} f_{2} f_{3}, H_{2}=g_{1}+g_{2}+g_{3}$ are functions of 3 variables with 24 parameters, 3 of them are essential. Without loss of generality, after removing the redundancy of the parameters, the invariants $H_{1}, H_{2}$ can be cast into the form

$$
H_{1}=\frac{x_{1}-p_{1}}{x_{1}} \frac{x_{2}-p_{2}}{x_{2}} \frac{x_{3}-p_{3}}{x_{3}}, \quad H_{2}=x_{1}+x_{2}+x_{3} .
$$

Then each of the mappings $R_{i j}, i \neq j \in\{1,2,3\}$ is exactly the $H_{\mathrm{II}}$ Yang-Baxter map.

For $k>3$ the invariants depend on $8 k$ parameters and only $3 k-6$ of them are essential. Without loss of generality they can be cast into the form

$$
H_{1}=\frac{x_{1}-p_{1}}{x_{1}} \frac{x_{2}-p_{2}}{x_{2}} \frac{x_{3}-p_{3}}{x_{3}} \prod_{i=4}^{k} \frac{\alpha_{i}-\beta_{i} x_{i}}{\beta_{i}-\gamma_{i} x_{i}}, \quad H_{2}=\sum_{i=1}^{k} x_{i} .
$$

\subsection{Additive/additive separability of variables}

Proposition 3.11. Consider the additive/additive separability of variables of the invariants $H_{1}$ and $\mathrm{H}_{2}$ (see (3.3)). Consider also the following sets of parameters

$$
\mathbf{p}_{i j}:=\mathbf{p}_{i} \cup \mathbf{p}_{j}, \quad \text { where } \quad \mathbf{p}_{i}:=\left\{a_{i}, b_{i}, c_{i}, d_{i}, A_{i}, B_{i}, C_{i}, D_{i}\right\}, \quad i \neq j<j \in\{1,2, \ldots, k\}
$$

and the functions

$$
f_{i}:=\frac{a_{i}-b_{i} x_{i}}{c_{i}-d_{i} x_{i}}, \quad g_{i}:=\frac{A_{i}-B_{i} x_{i}}{C_{i}-D_{i} x_{i}}, \quad i=1, \ldots, k .
$$

The following holds:

1. The invariants $H_{1}=\prod_{i=1}^{k} f_{i}, H_{2}=\sum_{i=1}^{k} g_{i}$ depend on $8 k$ parameters. Only $3 k-6$ of them are essential.

2. Mappings $R_{i j}$ explicitly read

$$
R_{i j}:\left(x_{1}, x_{2}, \ldots, x_{k}\right) \mapsto\left(X_{1}, X_{2}, \ldots, X_{k}\right),
$$


where $X_{l}=x_{l} \forall l \neq i, j$ and $X_{i}, X_{j}$ are given by the formulae

$$
X_{i}=x_{i}-2 \frac{\left|\begin{array}{ll}
f_{j}^{\prime} & f_{i}^{\prime} \\
g_{j}^{\prime} & g_{i}^{\prime}
\end{array}\right|}{\left|\begin{array}{ccc}
f_{i}^{\prime} & g_{i}^{\prime} \\
\frac{f_{j}^{\prime}}{f_{i}^{\prime}} f_{i}^{\prime \prime}+\frac{f_{i}^{\prime}}{f_{j}^{\prime}} f_{j}^{\prime \prime} & \frac{g_{j}^{\prime}}{g_{i}^{\prime}} g_{i}^{\prime \prime}+\frac{g_{i}^{\prime}}{g_{j}^{\prime}} g_{j}^{\prime \prime}
\end{array}\right|},
$$

where $f_{l}^{\prime} \equiv \frac{\partial f_{l}}{\partial x_{l}}, g_{l}^{\prime} \equiv \frac{\partial g_{l}}{\partial x_{l}}, g_{l}^{\prime \prime} \equiv \frac{\partial^{2} g_{l}}{\partial x_{l}^{2}}$, etc. Note that in the expressions of $X_{i}, X_{j}$ appears only the coordinates $x_{i}, x_{j}$ and the parameters $\mathbf{p}_{i j}$. From further on we denote the maps $R_{i j}$ as $R_{i j}^{\mathbf{p}_{i j}}$, in order to stress this separability feature.

3. Mappings $R_{i j}^{\mathbf{p}_{i j}}$ are anti-measure preserving with densities $m_{1}=n^{1} d^{2}, m_{2}=n^{2} d^{1}$, where $n^{i}, d^{i}$ the numerators and the denominators respectively, of the invariants $H_{i}, i=1,2$.

4. Mappings $R_{i j}^{\mathbf{p}_{i j}}$ satisfy the Yang-Baxter identity

$$
R_{i j}^{\mathbf{p}_{i j}} R_{i k}^{\mathbf{p}_{i k}} R_{j k}^{\mathbf{p}_{j k}}=R_{j k}^{\mathbf{p}_{j k}} R_{i j}^{\mathbf{p}_{i j}} R_{i j}^{\mathbf{p}_{i j}} .
$$

5. Mappings $R_{i j}^{\mathbf{p}_{i j}}$ are involutions with the sets of singularities

$$
\Sigma_{i j}=\left\{P_{i j}^{1}, P_{i j}^{2}\right\}=\left\{\left(\frac{c_{i}}{d_{i}}, \frac{c_{j}}{d_{j}}\right)^{2},\left(\frac{C_{i}}{D_{i}}, \frac{C_{j}}{D_{j}}\right)^{2}\right\},
$$

where the superscript 2 in $P_{i j}^{1}$ and $P_{i j}^{2}$ denotes that these singular points appears with multiplicity 2. In the formulae for $P_{i j}^{m}, m=1, \ldots, 2$, we have suppressed the dependency on the remaining variables. For example, with $P_{i j}^{1}=\left(\frac{c_{i}}{d_{i}}, \frac{c_{j}}{d_{j}}\right)$ we denote $\left(x_{1}, \ldots, x_{i-1}, \frac{c_{i}}{d_{i}}, x_{i+1}, \ldots\right.$, $\left.x_{j-1}, \frac{c_{j}}{d_{j}}, x_{j+1}, \ldots, x_{k}\right)$ and similarly for $P_{i j}^{2}$.

6. Each one of the maps $R_{i j}^{\mathbf{p}_{i j}}$ is (Möb) ${ }^{2}$ equivalent to the $H_{\mathrm{III}}^{A}$ Yang-Baxter map.

Proof. The proof follows similarly to the proof of Proposition 3.3.

Example $3.12(k \geq 3)$. For $k=3$, the invariants $H_{1}=f_{1}+f_{2}+f_{3}, H_{2}=g_{1}+g_{2}+g_{3}$ are functions of 3 variables with 24 parameters, 3 of them are essential. Without loss of generality, after removing the redundancy of the parameters, the invariants $H_{1}, H_{2}$ can be cast into the form:

$$
H_{1}=\frac{1}{x_{1}}+\frac{1}{x_{2}}+\frac{1}{x_{3}}, \quad H_{2}=p_{1} x_{1}+p_{2} x_{2}+p_{3} x_{3} .
$$

Then each of the mappings $R_{i j}, i \neq j \in\{1,2,3\}$ is exactly the $H_{\mathrm{III}}^{A}$ Yang-Baxter map.

For $k>3$ the invariants depend on $8 k$ parameters and only $3 k-6$ of them are essential. Without loss of generality they can be cast into the form

$$
H_{1}=\sum_{i=1}^{k} \frac{1}{x_{i}}, \quad H_{2}=p_{1} x_{1}+p_{2} x_{2}+p_{3} x_{3}+\sum_{i=4}^{k} \frac{\alpha_{i}-\beta_{i} x_{i}}{\beta_{i}-\gamma_{i} x_{i}} .
$$




\section{Entwining Yang-Baxter maps}

Following [51], three different maps $S, T, U$ are called entwining Yang-Baxter maps if they satisfy

$$
S_{12} T_{13} U_{23}=U_{23} T_{13} S_{12} .
$$

We consider two maps to be different if they are not (Möb) ${ }^{2}$ equivalent. Hence, in order to ensure that we have different maps we require that at least one of the maps $S, T, U$ either belongs to a different subclass than the remaining ones or it has different singularity pattern (even if it belongs to the same subclass with the remaining ones) or it has different periodicity. In what follows we present two methods to obtain entwining maps. The first one is based on degeneracy, i.e., we construct maps which belong to different subclasses and we obtain entwining maps associated with the $H_{\mathrm{I}}, H_{\mathrm{II}}$ and $H_{\mathrm{III}}^{A}$ families of maps. The second one is based on the symmetries of the $H$-list of Yang-Baxter maps and we obtain entwining maps for all members of the $H$-list.

\subsection{Degeneracy and entwining Yang-Baxter maps}

In Section 3.1 it was shown that for $k=3$ and for the multiplicative/multiplicative case, the invariants $H_{1}, H_{2}$ depend on 3 essential parameters. Without loss of generality they read

$$
H_{1}=x_{1} x_{2} x_{3}, \quad H_{2}=\frac{x_{1}-p_{1}}{x_{1}-1} \frac{x_{2}-p_{2}}{x_{2}-1} \frac{x_{3}-p_{3}}{x_{3}-1} .
$$

The associated maps $R_{12}, R_{13}$ and $R_{23}$ which preserve the invariants have exactly the form of the $H_{\mathrm{I}}$ map. In order to obtain entwining maps associated with the $H_{\mathrm{I}}$ map, we consider

$$
H_{1}=x_{1} x_{2} x_{3}, \quad H_{2}=\frac{x_{1}-p_{1}}{x_{1}-1} \frac{x_{2}-p_{2}}{x_{2}-1} \frac{\alpha_{3}-\beta_{3} x_{3}}{\beta_{3}-\gamma_{3} x_{3}} .
$$

For these invariants, $R_{12}$ is exactly the $H_{\mathrm{I}}$ map and for generic $\alpha_{3}, \beta_{3}, \gamma_{3}$ mappings $R_{13}$ and $R_{23}$ are $(\mathrm{Möb})^{2}$ equivalent to the $H_{\mathrm{I}}$. In order to obtain entwining maps we need to violate this (Möb) ${ }^{2}$ equivalency of the maps $R_{13}$ and $R_{23}$ with the $H_{\mathrm{I}}$ map. This is achieved by violating the generality, e.g., setting $\alpha_{3}=0$ or $\beta_{3}=0$, the maps $R_{13}$ and $R_{23}$, belongs to different subclasses than the $H_{\text {I }}$ map does. Working similarly for the $H_{\text {II }}$ map we find 1 family of maps which entwine with the latter without being (Möb) ${ }^{2}$ equivalent. Finally, for $H_{\mathrm{III}}^{A}$ we find also 1 family of entwining maps which are not (Möb $)^{2}$ equivalent with the latter. Our results are presented in Propositions 4.1-4.3.

Table 1. Entwining maps associated with the $H_{\mathrm{I}}$ Yang-Baxter map through degeneracy.

\begin{tabular}{c|c|l}
\hline map & $(u, v) \mapsto(U, V)$ & subclass \\
\hline $\mathrm{e}^{a} H_{\mathrm{I}}$ & $U=\frac{\alpha(1-u)+\beta(\alpha-1) u v}{\alpha-u}, V=\frac{u v(\alpha-u)}{\alpha(1-u)+\beta(\alpha-1) u v}$ & {$[1: 2]$} \\
$\mathrm{e}^{b} H_{\mathrm{I}}$ & $U=\frac{u-\alpha}{u-1}, V=\frac{u v(u-1)}{u-\alpha}$ & {$[0: 2]$} \\
\hline
\end{tabular}

Proposition 4.1. The $H_{\mathrm{I}}$ Yang-Baxter map entwines with the maps $\mathrm{e}^{a} H_{\mathrm{I}}$ and $\mathrm{e}^{b} H_{\mathrm{I}}$ of Table 1 according to the entwining relation

$$
S_{12} T_{13} T_{23}=T_{23} T_{13} S_{12}
$$

where $S_{12}$ is the $H_{\mathrm{I}}$ map acting on the $(1,2)$-coordinates, $T_{13}$ and $T_{23}$ are $\mathrm{e}^{a} H_{\mathrm{I}}$ acting on $(1,3)$ and $(2,3)$ coordinates respectively, or $\mathrm{e}^{b} H_{\mathrm{I}}$ acting on $(1,3)$ and $(2,3)$ coordinates respectively. 
Proof. Starting with the invariants

$$
H_{1}=x_{1} x_{2} x_{3}, \quad H_{2}=\frac{x_{1}-p_{1}}{x_{1}-1} \frac{x_{2}-p_{2}}{x_{2}-1} \frac{a-b x_{3}}{b-c x_{3}},
$$

the map $R_{12}$ is exactly the $H_{\mathrm{I}}$ map. By setting $a=0, R_{13}$ and $R_{23}$ takes the form of $\mathrm{e}^{a} H_{\mathrm{I}}$ of Table 1 (where $\beta \equiv c / b$ ). The map e ${ }^{a} H_{\mathrm{I}}$ is of subclass $[1: 2]$ so clearly non-(Möb ${ }^{2}$ equivalent to $H_{\mathrm{I}}$. By setting $b=0, R_{13}$ and $R_{23}$ takes the form of $\mathrm{e}^{b} H_{\mathrm{I}}$ of Table 1 (where $\beta \equiv a / c$ ). The map $\mathrm{e}^{b} H_{\mathrm{I}}$ is of subclass $[0: 1]$ so clearly non- $(\mathrm{Möb})^{2}$ equivalent to $H_{\mathrm{I}}$ or to $\mathrm{e}^{a} H_{\mathrm{I}}$. Finally, by setting $c=0$, mappings $R_{13}$ and $R_{23}$ are (Möb) ${ }^{2}$ equivalent to $\mathrm{e}^{a} H_{\mathrm{I}}$.

Proposition 4.2. The $H_{\mathrm{II}}$ Yang-Baxter map entwines with the map of Table 2 according to the entwining relation

$$
S_{12} T_{13} T_{23}=T_{23} T_{13} S_{12}
$$

where $S_{12}$ is the $H_{\mathrm{II}}$ map acting on the $(1,2)$-coordinates, $T_{13}$ and $T_{23}$ are $\mathrm{e}^{b} H_{\mathrm{II}}$ acting on $(1,3)$ and $(2,3)$ coordinates respectively.

Table 2. Entwining maps associated with the $H_{\text {II }}$ Yang-Baxter map though degeneracy.

\begin{tabular}{c|c|c}
\hline map & $(u, v) \mapsto(U, V)$ & subclass \\
\hline $\mathrm{e}^{b} H_{\mathrm{II}}$ & $U=\frac{\alpha v}{\alpha-u}, V=u \frac{\alpha-u-v}{\alpha-u}$ & {$[1: 1]$} \\
\hline
\end{tabular}

Proof. Starting with the invariants

$$
H_{1}=x_{1}+x_{2}+x_{3}, \quad H_{2}=\frac{x_{1}-p_{1}}{x_{1}} \frac{x_{2}-p_{2}}{x_{2}} \frac{a-b x_{3}}{b-c x_{3}},
$$

the map $R_{12}$ is exactly the $H_{\mathrm{II}}$ map. By setting $a=0, R_{13}$ and $R_{23}$ are (Möb) ${ }^{2}$ equivalent to the $H_{\text {II }}$ map. By setting $b=0, R_{13}$ and $R_{23}$ takes the form of $\mathrm{e}^{b} H_{\text {II }}$ of Table 2 . The map e $\mathrm{e}^{b} H_{\mathrm{II}}$ is of subclass $[1: 1]$ so clearly non-(Möb $)^{2}$ equivalent to the $H_{\mathrm{II}}$ map. Finally, by setting $c=0$, mappings $R_{13}$ and $R_{23}$ are (Möb) ${ }^{2}$ equivalent to $\mathrm{e}^{b} H_{\mathrm{II}}$.

Proposition 4.3. The $H_{\mathrm{III}}^{A}$ Yang-Baxter map entwines with the map of Table 3 according to the entwining relation

$$
S_{12} T_{13} T_{23}=T_{23} T_{13} S_{12}
$$

where $S_{12}$ is the $H_{\mathrm{III}}^{A}$ map acting on the $(1,2)$-coordinates, $T_{13}$ and $T_{23}$ are $\mathrm{e}^{b} H_{\mathrm{III}}^{A}$ acting on $(1,3)$ and $(2,3)$ coordinates respectively.

Table 3. Entwining maps associated with the $H_{\mathrm{III}}^{A}$ Yang-Baxter map though degeneracy.

\begin{tabular}{c|c|c}
\hline map & $(u, v) \mapsto(U, V)$ & subclass \\
\hline $\mathrm{e}^{b} H_{\mathrm{III}}^{A}$ & $U=\frac{\beta}{\alpha} u, V=\frac{\beta u v}{\beta(u+v)-\alpha u^{2} v}$ & {$[0: 2]$} \\
\hline
\end{tabular}


Proof. Starting with the invariants

$$
H_{1}=x_{1}+x_{2}+x_{3}, \quad H_{2}=p_{1} x_{1}+p_{2} x_{2}+\frac{a-b x_{3}}{b-c x_{3}},
$$

the map $R_{12}$ is exactly the $H_{\mathrm{III}}^{A}$ map. By setting $a=0, R_{13}$ and $R_{23}$ are (Möb) ${ }^{2}$ equivalent to the $H_{\mathrm{III}}^{A}$ map. By setting $b=0$ and $R_{13}$ and $R_{23}$ takes the form of $\mathrm{e}^{b} H_{\mathrm{III}}^{A}$ of Table 3 (where $\beta=a / c)$. The map $\mathrm{e}^{b} H_{\mathrm{III}}^{A}$ is of subclass $[0: 2]$ so clearly non-(Möb) ${ }^{2}$ equivalent to the $H_{\mathrm{III}}^{A}$ map. Finally, by setting $c=0$, mappings $R_{13}$ and $R_{23}$ are (Möb) $)^{2}$ equivalent to the $H_{\mathrm{III}}^{A}$ map.

In the following subsection we are using the notion of symmetry of Yang-Baxter maps in order to generate entwining maps

\subsection{Symmetries of Yang-Baxter maps and the entwining property}

The notion of symmetry in the context of Yang-Baxter maps was introduced in [59].

Definition 4.4. An involution $\phi: \mathbb{C P}^{1} \mapsto \mathbb{C P}^{1}$ is a symmetry of the Yang-Baxter map $R: \mathbb{C P}^{1} \times$ $\mathbb{C P}^{1} \mapsto \mathbb{C P}^{1} \times \mathbb{C P}^{1}$ if it holds

$$
\phi_{1} \phi_{2} R_{12}=R_{12} \phi_{1} \phi_{2}
$$

where $\phi_{1}$ is the involution that acts as $\phi$ to the first factor of the cartesian product $\mathbb{C P}^{1} \times \mathbb{C P}^{1}$ and $\phi_{2}$ is the involution that acts as $\phi$ to the second factor of the cartesian product.

Let $m<n \in\{1, \ldots, k\}, k \geq 3$ fixed. A direct consequence of the previous definition is that if $\phi$ is a symmetry of the Yang-Baxter map $R$, then the map $\phi_{m} R_{m n} \phi_{n}$ is a new Yang-Baxter map since it is not (Möb) ${ }^{2}$ equivalent with $R_{m n}$. By finding the symmetries of the $F$-list of Yang-Baxter maps, the authors of [59] derived the $H$-list of Yang-Baxter maps. Clearly the symmetries of the $F$-list are symmetries of the $H$-list and vice versa.

Theorem 4.5. Let $\phi$ a symmetry of a Yang-Baxter map $R$ and let $\phi_{0}$ the identity map, i.e., $\phi_{0}:\left(x_{1}, \ldots, x_{k}\right) \mapsto\left(x_{1}, \ldots, x_{k}\right)$. Out of the possible $4^{3}$ entwining relations of the form

$$
R_{12} \phi_{i} R_{13} \phi_{j} R_{23} \phi_{k}=R_{23} \phi_{k} R_{13} \phi_{j} R_{12} \phi_{i}, \quad i, j, k \in\{0,1,2,3\},
$$

apart the Yang-Baxter relation that holds, only the following three entwining relations holds

$$
\begin{aligned}
& R_{12} R_{13} \phi_{1} R_{23} \phi_{2}=R_{23} \phi_{2} R_{13} \phi_{1} R_{12}, \\
& R_{12} \phi_{2} R_{13} \phi_{3} R_{23}=R_{23} R_{13} \phi_{3} R_{12} \phi_{2}, \\
& R_{12} \phi_{2} R_{13} \phi_{2} R_{23} \phi_{2}=R_{23} \phi_{2} R_{13} \phi_{2} R_{12} \phi_{2} .
\end{aligned}
$$

Proof. To show that only the entwining relations (4.2), (4.3), (4.4) holds, we start with

$$
R_{12} \phi_{i} R_{13} \phi_{j} R_{23} \phi_{k}=R_{23} \phi_{k} R_{13} \phi_{j} R_{12} \phi_{i}, \quad i, j, k \in\{0,1,2,3\} .
$$

By direct calculations, we prove that if the Yang-Baxter relation holds out of the $4^{3}$ different relations (4.1), only (4.2), (4.3), (4.4) holds.

For example let us show that (4.2) holds. We have

$$
R_{12} R_{13} \phi_{1} R_{23} \phi_{2}=R_{12} R_{13} R_{23} \phi_{1} \phi_{2}=R_{23} R_{13} R_{12} \phi_{1} \phi_{2},
$$

since $\phi_{1}$ commutes with $R_{23}$ and the Yang-Baxter relation $R_{12} R_{13} R_{23}=R_{23} R_{13} R_{12}$ holds. But due to the symmetry we have $R_{12} \phi_{1} \phi_{2}=\phi_{1} \phi_{2} R_{12}$ so (4.5) reads

$$
R_{23} R_{13} R_{12} \phi_{1} \phi_{2}=R_{23} R_{13} \phi_{1} \phi_{2} R_{12}=R_{23} \phi_{2} R_{13} \phi_{1} R_{12}
$$

and that completes the proof that (4.2) holds. For the remaining relations we work similarly for their proof. 
Note that any of the entwining relations (4.2), (4.3) and (4.4), is uniquely described by the symmetries $\phi_{i}, \phi_{j}, \phi_{k}$ that take part in this relation. For example in (4.2) the symmetries $\phi_{0}$, $\phi_{1}, \phi_{2}$ appear in this order, hence we refer to (4.2) as relation of entwining type $\left(\phi_{0}, \phi_{1}, \phi_{2}\right)$ or by using just the subscripts, relation of entwining type $(0,1,2)$.

In Table 4, we present the entwining maps $S, T, U$ that correspond to the entwining relations (4.2)-(4.4), where $R$ is any Yang-Baxter map. In what follows, we specify $R$ to be any member of the $H$-list ${ }^{2}$ of quadrirational Yang-Baxter maps.

Table 4. Entwining maps $S, T, U$ associated with a Yang-Baxter map $R$.

\begin{tabular}{c|c|c|c}
\hline entwining type & $S_{12}$ & $T_{13}$ & $U_{23}$ \\
\hline$(0,1,2)$ & $R_{12}$ & $R_{13} \phi_{1}$ & $R_{23} \phi_{2}$ \\
\hline$(2,3,0)$ & $R_{12} \phi_{2}$ & $R_{13} \phi_{3}$ & $R_{23}$ \\
\hline$(2,2,2)$ & $R_{12} \phi_{2}$ & $R_{13}$ & $\phi_{2} R_{23} \phi_{2}$ \\
\hline \hline
\end{tabular}

\subsubsection{Entwining maps associated with the $H_{\mathrm{I}}$ Yang-Baxter map}

The involutions $\phi, \psi$

$$
\phi: u \mapsto \frac{\alpha}{u}, \quad \psi: u \mapsto \frac{u-\alpha}{u-1},
$$

where $\alpha$ a complex parameter, are symmetries for the $H_{\text {I }}$ map (see [59]), since it holds

$$
\phi_{1} \phi_{2} R_{12}=R_{12} \phi_{1} \phi_{2}, \quad \psi_{1} \psi_{2} R_{12}=R_{12} \psi_{1} \psi_{2}
$$

where $R_{12}$ is the $H_{\mathrm{I}}$ map acting on the 12 -coordinates and

$$
\begin{array}{llll}
\phi_{1}: & \left(x_{1}, x_{2}\right) & \mapsto\left(p_{1} / x_{1}, x_{2}\right), & \phi_{2}:\left(x_{1}, x_{2}\right) \mapsto\left(x_{1}, p_{2} / x_{2}\right), \\
\psi_{1}: & \left(x_{1}, x_{2}\right) \mapsto\left(\left(x_{1}-p_{1}\right) /\left(x_{1}-1\right), x_{2}\right), & \psi_{2}: & \left(x_{1}, x_{2}\right) \mapsto\left(x_{1},\left(x_{2}-p_{2}\right) /\left(x_{2}-1\right)\right) .
\end{array}
$$

Note that the symmetries $\phi$ and $\tau$ can be derived from our considerations (see Example 3.6) since for $k=3$ it holds

$$
\begin{aligned}
H_{1} \phi_{1} \phi_{2} \phi_{3} & =\frac{p_{1} p_{2} p_{3}}{H_{1}}, & H_{2} \phi_{1} \phi_{2} \phi_{3} & =\frac{1}{H_{2}}, \\
H_{1} \psi_{1} \psi_{2} \psi_{3} & =H_{2}, & H_{2} \psi_{1} \psi_{2} \psi_{3} & =H_{1} .
\end{aligned}
$$

Remark 4.6. By using similar arguments as in the proof of the Theorem 4.5, entwining relations where the symmetries $\phi$ and $\psi$ of the $H_{\mathrm{I}}$ map interlace do not exist, i.e., it does not exists for example any relation of entwining type $\left(\phi_{i}, \phi_{j}, \psi_{k}\right)$.

In Table 5 we present the entwining maps associated with the $H_{\text {I }}$ map which are generated by using the symmetries $\phi$ and $\psi$. In Table 5 it appears the $H_{\mathrm{I}}$ map, the companion of the $H_{\mathrm{I}}$ map that is denoted as $c H_{\mathrm{I}}$, as well as $\tilde{c} F_{\mathrm{I}}$ which is the companion map of the map $\tilde{F}_{\mathrm{I}}$ that was derived in [59]. We also have four novel maps which are not (Möb) ${ }^{2}$ equivalent to $H_{\mathrm{I}}$, which we refer to as $\Phi_{\mathrm{I}}^{a}, \Phi_{\mathrm{I}}^{b}, \Psi_{\mathrm{I}}^{a}$ and $\Psi_{\mathrm{I}}^{b}$. In the proposition that follows we present their explicit form.

\footnotetext{
${ }^{2}$ It is easy to show that the entwining maps associated with the $F$-list of quadrirational Yang-Baxter maps are $(\mathrm{Möb})^{2}$ equivalent to the corresponding to the $H$-list entwining maps. This is the reason that we present the entwining maps associated with the $H$-list only.
} 
Table 5. Left table: Entwining maps $S, T, U$ associated with $H_{\mathrm{I}}$ Yang-Baxter map using the symmetry $\phi$. Right table: Entwining maps $S, T, U$ associated with $H_{\text {I }}$ Yang-Baxter map using the symmetry $\psi$.

\begin{tabular}{c|c|c|c}
\hline entwining type & $S_{12}$ & $T_{13}$ & $U_{23}$ \\
\hline$(0,1,2)$ & $H_{\mathrm{I}}$ & $\Phi_{\mathrm{I}}^{a}$ & $\Phi_{\mathrm{I}}^{a}$ \\
\hline$(2,3,0)$ & $\Phi_{\mathrm{I}}^{b}$ & $\Phi_{\mathrm{I}}^{b}$ & $H_{\mathrm{I}}$ \\
\hline$(2,2,2)$ & $\Phi_{\mathrm{I}}^{b}$ & $H_{\mathrm{I}}$ & $c H_{\mathrm{I}}$ \\
\hline \hline
\end{tabular}

\begin{tabular}{c|c|c|c}
\hline entwining type & $S_{12}$ & $T_{13}$ & $U_{23}$ \\
\hline$(0,1,2)$ & $H_{\mathrm{I}}$ & $\Psi_{\mathrm{I}}^{a}$ & $\Psi_{\mathrm{I}}^{a}$ \\
\hline$(2,3,0)$ & $\Psi_{\mathrm{I}}^{b}$ & $\Psi_{\mathrm{I}}^{b}$ & $H_{\mathrm{I}}$ \\
\hline$(2,2,2)$ & $\Psi_{\mathrm{I}}^{b}$ & $H_{\mathrm{I}}$ & $c \tilde{F}_{\mathrm{I}}$ \\
\hline \hline
\end{tabular}

Proposition 4.7. The following non-periodic ${ }^{3}$ maps $(u, v) \mapsto(U, V)$, where

$$
\begin{aligned}
& U=\alpha v Q, \quad V=\frac{1}{u} Q^{-1}, \quad Q=\frac{\beta-\alpha+u(1-\beta)+v(\alpha-1)}{\beta(1-\alpha) u-\alpha(1-\beta) v+(\alpha-\beta) u v} \\
& U=\frac{1}{v} Q^{-1}, \quad V=\beta u Q, \quad Q=\frac{\beta-\alpha+u(1-\beta)+v(\alpha-1)}{\beta(1-\alpha) u-\alpha(1-\beta) v+(\alpha-\beta) u v} \\
& U=v Q, \quad V=\frac{u-\alpha}{u-1} Q^{-1}, \quad Q=\frac{\alpha(1-v)-\beta u+u v}{\beta(1-u)-\beta v+u v}, \\
& U=\frac{v-\beta}{v-1} Q, \quad V=u Q^{-1}, \quad Q=\frac{\alpha(1-u-v)+u v}{\beta(1-u)-\alpha v+u v},
\end{aligned}
$$

entwine with the $H_{\mathrm{I}}$ Yang-Baxter map according to the entwining relations of Table 5.

\subsubsection{Entwining maps associated with the $H_{\text {II }}$ Yang-Baxter map}

The invariants

$$
H_{1}=x_{1}+x_{2}+x_{3}, \quad H_{2}=\frac{x_{1}-p_{1}}{x_{1}} \frac{x_{2}-p_{2}}{x_{2}} \frac{x_{3}-p_{3}}{x_{3}},
$$

generate the maps $R_{i j}, i<j \in\{1,2,3\}$ which are exactly the $H_{\mathrm{II}}$ map acting on the $(i j)$ coordinates. Explicitly the $H_{\mathrm{II}}$ map reads

$$
U=v+\frac{(\alpha-\beta) u v}{\beta u+\alpha v-\alpha \beta}, \quad V=u-\frac{(\alpha-\beta) u v}{\beta u+\alpha v-\alpha \beta} .
$$

A symmetry of the $H_{\text {II }}$ map is $\phi: u \mapsto \alpha-u$, since it holds $\phi_{1} \phi_{2} R_{12}=R_{12} \phi_{1} \phi_{2}$, where $R_{12}$ is the $H_{\mathrm{II}}$ map acting on the (12)-coordinates and

$$
\phi_{1}:\left(x_{1}, x_{2}\right) \mapsto\left(p_{1}-x_{1}, x_{2}\right), \quad \phi_{2}:\left(x_{1}, x_{2}\right) \mapsto\left(x_{1}, p_{2}-x_{2}\right)
$$

Table 6. Entwining maps $S, T, U$ associated with $H_{\mathrm{II}}$ Yang-Baxter map using the symmetry $\phi$.

\begin{tabular}{c|c|c|c}
\hline entwining type & $S_{12}$ & $T_{13}$ & $U_{23}$ \\
\hline$(0,1,2)$ & $H_{\mathrm{II}}$ & $\Phi_{\mathrm{II}}^{a}$ & $\Phi_{\mathrm{II}}^{a}$ \\
\hline$(2,3,0)$ & $\Phi_{\mathrm{II}}^{b}$ & $\Phi_{\mathrm{II}}^{b}$ & $H_{\mathrm{II}}$ \\
\hline$(2,2,2)$ & $\Phi_{\mathrm{II}}^{b}$ & $H_{\mathrm{II}}$ & $c H_{\mathrm{II}}$ \\
\hline \hline
\end{tabular}

\footnotetext{
${ }^{3}$ A non-periodic map cannot be equivalent by conjugation ((Möb $)^{2}$ equivalent) to a periodic map. Since the $H_{\mathrm{I}}$ map is involutive, the maps presented in this proposition are not (Möb) ${ }^{2}$ to the $H_{\mathrm{I}}$ map.
} 
Proposition 4.8. The following non-periodic maps $(u, v) \mapsto(U, V)$, where

$$
\begin{array}{ll}
U=\alpha v \frac{u-v+\beta-\alpha}{\beta u-\alpha v}, & V=\beta \frac{(\alpha-u)(u-v)}{\beta u-\alpha v}, \\
U=\alpha \frac{(\beta-v)(u-v)}{\beta u-\alpha v}, & V=\beta u \frac{u-v+\beta-\alpha}{\beta u-\alpha v},
\end{array}
$$

entwine with the $H_{\mathrm{II}}$ Yang-Baxter map according to the entwining relations of Table 6.

The map $c H_{\mathrm{II}}$ denotes the companion map of the $H_{\mathrm{II}}$ map.

\subsubsection{Entwining maps associated with the $H_{\text {III }}^{A}$ Yang-Baxter map}

The invariants

$$
H_{1}=\frac{1}{x_{1}}+\frac{1}{x_{2}}+\frac{1}{x_{3}}, \quad H_{2}=p_{1} x_{1}+p_{2} x_{2}+p_{3} x_{3},
$$

generate the maps $R_{i j}, i<j \in\{1,2,3\}$ which are exactly the $H_{\text {III }}^{A}$ map acting on the $(i j)$ coordinates. Explicitly the $H_{\mathrm{III}}^{A}$ map reads

$$
U=\frac{v}{\alpha} \frac{\alpha u+\beta v}{u+v}, \quad V=\frac{u}{\beta} \frac{\alpha u+\beta v}{u+v} .
$$

Two symmetries of the $H_{\mathrm{III}}^{A}$ map are

$$
\phi: u \mapsto \frac{1}{\alpha u}, \quad \psi: u \mapsto-u
$$

since it holds

$$
\phi_{1} \phi_{2} R_{12}=R_{12} \phi_{1} \phi_{2}, \quad \psi_{1} \psi_{2} R_{12}=R_{12} \psi_{1} \psi_{2},
$$

where $R_{12}$ is the $H_{\mathrm{III}}^{A}$ map acting on the (12)-coordinates and

$$
\begin{array}{rlrl}
\phi_{1}:\left(x_{1}, x_{2}\right) & \mapsto\left(\frac{1}{p_{1} x_{1}}, x_{2}\right), & \phi_{2}:\left(x_{1}, x_{2}\right) & \mapsto\left(x_{1}, \frac{1}{p_{2} x_{2}}\right), \\
\psi_{1}:\left(x_{1}, x_{2}\right) & \mapsto\left(-x_{1}, x_{2}\right), & \psi_{2}:\left(x_{1}, x_{2}\right) \mapsto\left(x_{1},-x_{2}\right) .
\end{array}
$$

Note that the map $\phi_{1} R_{12} \phi_{2}$ is exactly the $H_{\mathrm{III}}^{B}$ Yang-Baxter map.

Proposition 4.9. The following non-periodic maps $(u, v) \mapsto(U, V)$ where

$$
\begin{aligned}
U & =v \frac{1+\beta u v}{1+\alpha u v}, & V & =\frac{1}{\beta u} \frac{1+\beta u v}{1+\alpha u v}, \\
U & =\frac{1}{\alpha v} \frac{1+\alpha u v}{1+\beta u v}, & V & =u \frac{1+\alpha u v}{1+\beta u v} \\
U & =\frac{v}{\alpha} \frac{\alpha u-\beta v}{u-v}, & V & =\frac{u}{\beta} \frac{\alpha u-\beta v}{v-u} \\
U & =\frac{v}{\alpha} \frac{\alpha u-\beta v}{v-u}, & V & =\frac{u}{\beta} \frac{\alpha u-\beta v}{u-v}
\end{aligned}
$$

entwine with the $H_{\mathrm{III}}^{A}$ Yang-Baxter map according to the entwining relations of Table 7.

The map $c H_{\mathrm{III}}^{A}$ denotes the companion map of the $H_{\mathrm{III}}^{A}$ map and with $\hat{H}_{\mathrm{III}}^{A}$ we denote a (Möb) ${ }^{2}$ equivalent map to the $H_{\mathrm{III}}^{A}$. 
Table 7. Left table: Entwining maps $S, T, U$ associated with $H_{\text {III }}^{A}$ Yang-Baxter map using the symmetry $\phi$. Right table: Entwining maps $S, T, U$ associated with $H_{\mathrm{III}}^{A}$ Yang-Baxter map using the symmetry $\psi$.

\begin{tabular}{c|c|c|c}
\hline entwining type & $S_{12}$ & $T_{13}$ & $U_{23}$ \\
\hline$(0,1,2)$ & $H_{\mathrm{III}}^{A}$ & $\Phi_{\mathrm{III}}^{a}$ & $\Phi_{\mathrm{III}^{A}}^{a}$ \\
\hline$(2,3,0)$ & $\Phi_{\mathrm{III}}^{b}$ & $\Phi_{\mathrm{III}}^{b}$ & $H_{\mathrm{III}}^{A}$ \\
\hline$(2,2,2)$ & $\Phi_{\mathrm{III}^{A}}^{b}$ & $H_{\mathrm{III}}^{A}$ & $\hat{H}_{\mathrm{III}}^{A}$ \\
\hline \hline
\end{tabular}

\begin{tabular}{c|c|c|c}
\hline entwining type & $S_{12}$ & $T_{13}$ & $U_{23}$ \\
\hline$(0,1,2)$ & $H_{\mathrm{III}}^{A}$ & $\Psi_{\mathrm{III}^{A}}^{a}$ & $\Psi_{\mathrm{III}}^{a}$ \\
\hline$(2,3,0)$ & $\Psi_{\mathrm{III}}^{b}$ & $\Psi_{\mathrm{III}^{A}}^{b}$ & $H_{\mathrm{III}}^{A}$ \\
\hline$(2,2,2)$ & $\Psi_{\mathrm{III}}^{b}$ & $H_{\mathrm{III}}^{A}$ & $c H_{\mathrm{III}}^{A}$ \\
\hline \hline
\end{tabular}

\subsubsection{Entwining maps associated with the $H_{\mathrm{III}}^{B}$ Yang-Baxter map}

The invariants that were derived in $[44,45,47,56]$,

$$
H_{1}=x_{1} x_{2} x_{3}, \quad H_{2}=p_{1} x_{1}+p_{2} x_{2}+p_{3} x_{3}+\frac{1}{x_{1}}+\frac{1}{x_{2}}+\frac{1}{x_{3}},
$$

generate the maps $R_{i j}, i<j \in\{1,2,3\}$ which are exactly the $H_{\mathrm{III}}^{B}$ map acting on the $(i j)$ coordinates. Explicitly the $H_{\mathrm{III}}^{B}$ map reads

$$
U=v \frac{1+\beta u v}{1+\alpha u v}, \quad V=u \frac{1+\alpha u v}{1+\beta u v},
$$

The symmetries $\phi, \psi$ of the $H_{\mathrm{III}}^{A}$ map are symmetries of $H_{\mathrm{III}}^{B}$ as well.

Proposition 4.10. The following non-periodic maps $(u, v) \mapsto(U, V)$, where

$$
\begin{aligned}
U & =\frac{v}{\alpha} \frac{\alpha u+\beta v}{u+v}, & V & =\frac{1}{u} \frac{u+v}{\alpha u+\beta v}, \\
U & =\frac{1}{v} \frac{u+v}{\alpha u+\beta v}, & V & =\frac{u}{\beta} \frac{\alpha u+\beta v}{u+v}, \\
U & =v \frac{1-\beta u v}{1-\alpha u v}, & V & =u \frac{1-\alpha u v}{-1+\beta u v} \\
U & =v \frac{1-\beta u v}{-1+\alpha u v}, & V & =u \frac{1-\alpha u v}{1-\beta u v}
\end{aligned}
$$

entwine with the $H_{\mathrm{III}}^{B}$ Yang-Baxter map according to the entwining relations of Table 8.

Table 8. Left table: Entwining maps $S, T, U$ associated with $H_{\mathrm{III}}^{B}$ Yang-Baxter map using the symmetry $\phi$. Right table: Entwining maps $S, T, U$ associated with $H_{\mathrm{III}}^{B}$ Yang-Baxter map using the symmetry $\psi$.

\begin{tabular}{c|c|c|c}
\hline entwining type & $S_{12}$ & $T_{13}$ & $U_{23}$ \\
\hline$(0,1,2)$ & $H_{\mathrm{III}}^{B}$ & $\Phi_{\mathrm{III}}^{a}$ & $\Phi_{\mathrm{III}^{B}}^{a}$ \\
\hline$(2,3,0)$ & $\Phi_{\mathrm{III}^{B}}^{b}$ & $\Phi_{\mathrm{III}}^{b}$ & $H_{\mathrm{III}}^{B}$ \\
\hline$(2,2,2)$ & $\Phi_{\mathrm{III}^{B}}^{b}$ & $H_{\mathrm{III}}^{B}$ & $\hat{H}_{\mathrm{III}}^{B}$ \\
\hline \hline
\end{tabular}

\begin{tabular}{c|c|c|c}
\hline entwining type & $S_{12}$ & $T_{13}$ & $U_{23}$ \\
\hline$(0,1,2)$ & $H_{\mathrm{III}}^{B}$ & $\Psi_{\mathrm{III}^{B}}^{a}$ & $\Psi_{\mathrm{III}}^{a}$ \\
\hline$(2,3,0)$ & $\Psi_{\mathrm{III}}^{b}$ & $\Psi_{\mathrm{III}^{B}}^{b}$ & $H_{\mathrm{III}}^{B}$ \\
\hline$(2,2,2)$ & $\Psi_{\mathrm{III}^{B}}^{b}$ & $H_{\mathrm{III}}^{B}$ & $\tilde{H}_{\mathrm{III}}^{B}$ \\
\hline \hline
\end{tabular}

The maps $\hat{H}_{\mathrm{III}}^{B}, \tilde{H}_{\mathrm{III}}^{B}$ that appear in Table 8 , are $(\mathrm{Möb})^{2}$ equivalent to the map $H_{\mathrm{III}}^{B}$. The map $c H_{\mathrm{III}}^{B}$ denotes the companion map of the $H_{\mathrm{III}}^{B}$ map.

\subsubsection{Entwining maps associated with the $H_{\mathrm{V}}$ Yang-Baxter map}

The invariants that were derived in $[44,45,47,56]$,

$$
H_{1}=x_{1}+x_{2}+x_{3}, \quad H_{2}=x_{1}^{3}+3 p_{1} x_{1}+x_{2}^{3}+3 p_{2} x_{2}+x_{3}^{3}+3 p_{3} x_{3},
$$


generate the maps $R_{i j}, i<j \in\{1,2,3\}$ which are exactly the $H_{\mathrm{V}}$ map acting on the $(i j)$ coordinates. Explicitly the $H_{\mathrm{V}}$ map reads

$$
U=v-\frac{\alpha-\beta}{u+v}, \quad V=u+\frac{\alpha-\beta}{u+v} .
$$

The involution $\psi: u \mapsto-u$ is a symmetry of the $H_{\mathrm{V}}$ map.

Proposition 4.11. The following non-periodic maps $(u, v) \mapsto(U, V)$, where

$$
\begin{array}{ll}
U=v+\frac{\alpha-\beta}{u-v}, & V=-u-\frac{\alpha-\beta}{u-v}, \\
U=-v-\frac{\alpha-\beta}{u-v}, & V=u+\frac{\alpha-\beta}{u-v},
\end{array}
$$

entwine with the $H_{\mathrm{V}}$ Yang-Baxter map according to the entwining relations of Table 9.

Table 9. Entwining maps $S, T, U$ associated with $H_{\mathrm{V}}$ Yang-Baxter map using the symmetry $\psi$.

\begin{tabular}{c|c|c|c}
\hline entwining type & $S_{12}$ & $T_{13}$ & $U_{23}$ \\
\hline$(0,1,2)$ & $H_{\mathrm{V}}$ & $\Psi_{\mathrm{V}}^{a}$ & $\Psi_{\mathrm{V}}^{a}$ \\
\hline$(2,3,0)$ & $\Psi_{\mathrm{V}}^{b}$ & $\Psi_{\mathrm{V}}^{b}$ & $H_{\mathrm{V}}$ \\
\hline$(2,2,2)$ & $\Psi_{\mathrm{V}}^{b}$ & $H_{\mathrm{V}}$ & $c H_{\mathrm{V}}$ \\
\hline \hline
\end{tabular}

The map $c H_{\mathrm{V}}$ denotes the companion map of the $H_{\mathrm{V}}$ map.

\section{$5 \quad$ Transfer maps}

The notion of transfer maps associated with Yang-Baxter maps was introduced by Veselov in [69]. In [70] dynamical aspects of the latter were discussed. The transfer maps associated with any reversible Yang-Baxter map are defined as

$$
T_{i}^{(k)}=R_{i i+k-1} R_{i i+k-2} \cdots R_{i i+1}, \quad i \in\{1, \ldots, k\},
$$

where the indices are considered modulo $k$. There is:

$$
T_{i}^{(k)} T_{j}^{(k)}=T_{j}^{(k)} T_{i}^{(k)}, \quad T_{1}^{(k)} T_{2}^{(k)} \cdots T_{k}^{(k)}=\mathrm{id} .
$$

For example for $k=4$ we have $T_{1}^{(4)}=R_{14} R_{13} R_{12}, T_{2}^{(4)}=R_{12} R_{24} R_{23}, T_{3}^{(4)}=R_{23} R_{13} R_{34}$ and $T_{4}^{(4)}=R_{34} R_{24} R_{14}$.

Proposition 5.1. For the transfer maps $T_{i}^{(k)}$ associated with the maps $R_{i j}^{\mathbf{p}_{i j}}$ of the Propositions 3.3, 3.9, 3.11, it holds:

1) they preserve the invariants $H_{1}, H_{2}$, presented in the Propositions 3.3, 3.9, 3.11,

2) for $k=2 n+1$ they preserve the measures given in the Propositions 3.3, 3.9, 3.11,

3) for $k=2 n$ they anti-preserve the measures given in the Propositions 3.3, 3.9, 3.11,

4) they possess Lax pairs,

5) for generic values of the parameter sets $\mathbf{p}_{i j}$, are equivalent by conjugation to the transfer maps associated with $H_{\mathrm{I}}, H_{\mathrm{II}}$ and $H_{\mathrm{III}}^{A}$ Yang-Baxter maps respectively, 
6) for non-generic values of the parameter sets $\mathbf{p}_{i j}$, we have novel transfer maps.

Proof. The statements (1)-(3) have already been proven (see Propositions 2.1, 3.3, 3.9, 3.11). As for the statement (4), one can construct a Lax matrix for the Yang-Baxter map $R$ following [66]. Then the Lax equations associated with the transfer maps $T_{i}^{(k)}$, correspond to certain factorizations of the monodromy matrix (see [69]).

We will show the statement (5) for the transfer maps associated with $R_{i j}^{\mathbf{p}_{i j}}$ of Proposition 3.3 and for $k=4$. The proof for arbitrary $k$ follows by induction. In Proposition 3.3 it was shown that these maps are (Möb) ${ }^{2}$ equivalent to the $H_{\text {I }}$ map. Let us denote as $\nu_{l}$ the maps defined by the cross-ratios

$$
\mathrm{CR}\left[x_{l}, a_{l} / b_{l}, c_{l} / d_{l}, A_{l} / B_{l}\right]=\mathrm{CR}\left[y_{l}, 0,1, \infty\right], \quad l=1, \ldots, 4,
$$

and as $\mu_{l}$ the maps defined by

$$
\mathrm{CR}\left[x_{l}, c_{l} / d_{l}, a_{l} / b_{l}, C_{l} / D_{l}\right]=\mathrm{CR}\left[y_{l}, \infty, 1,0\right], \quad l=1, \ldots, 4 .
$$

Then the maps $\tilde{R}_{i j}^{\mathbf{p}_{i j}}$, where $\tilde{R}_{i j}^{\mathbf{p}_{i j}}=\mu_{j}^{-1} \mu_{i}^{-1} R_{i j}^{\mathbf{p}_{i j}} \mu_{i} \mu_{j}$ are exactly the $H_{\text {I }}$ map acting on the (ij)-coordinates (see Proposition 3.3). For the transfer map $\tilde{T}_{1}^{(4)}$ associated with $\tilde{R}_{i j}^{\mathbf{p}_{i j}}$, there is

$$
\begin{aligned}
\tilde{T}_{1}^{(4)} & =\tilde{R}_{14} \tilde{R}_{13} \tilde{R}_{12}=\left(\nu_{1}^{-1} \mu_{4}^{-1} R_{14} \nu_{1} \mu_{4}\right)\left(\nu_{1}^{-1} \mu_{3}^{-1} R_{13} \nu_{1} \mu_{3}\right)\left(\nu_{1}^{-1} \mu_{3}^{-1} R_{13} \nu_{1} \mu_{2}\right) \\
& =\mu_{4}^{-1} \mu_{3}^{-1} \mu_{2}^{-1} \nu_{1}^{-1} R_{14} R_{13} R_{12} \nu_{1} \mu_{2} \mu_{3} \mu_{4}=\mu_{4}^{-1} \mu_{3}^{-1} \mu_{2}^{-1} \nu_{1}^{-1} T_{1}^{(4)} \nu_{1} \mu_{2} \mu_{3} \mu_{4} .
\end{aligned}
$$

Note that we have omitted the parameter sets $\mathbf{p}_{i j}$ that the maps depends on for simplicity.

(6). For non-generic choice of the parameter sets $\mathbf{p}_{i j}$, the conjugation equivalence (5.1) does not holds.

\subsection{On a re-factorisation of the transfer maps}

First, let us introduce some maps. With $\pi_{i j}$ we denote the transpositions

$$
\begin{aligned}
& \pi_{i j}:\left(x_{1}, \ldots, x_{k} ; \mathbf{p}_{1}, \ldots, \mathbf{p}_{k}\right) \mapsto\left(X_{1}, \ldots, X_{k} ; \mathbf{P}_{1}, \ldots, \mathbf{P}_{k}\right), \\
& X_{l}=x_{l}, \quad \mathbf{P}_{l}=\mathbf{p}_{l} \quad \forall l \neq i, j, \quad X_{i}=x_{j}, \quad X_{j}=x_{i}, \quad \mathbf{P}_{i}=\mathbf{p}_{j}, \quad \mathbf{P}_{j}=\mathbf{p}_{i} .
\end{aligned}
$$

and with $\pi_{0}$ we denote the following $k$-periodic map

$$
\begin{aligned}
& \pi_{0}:\left(x_{1}, \ldots, x_{k} ; \mathbf{p}_{1}, \ldots, \mathbf{p}_{k}\right) \mapsto\left(X_{1}, \ldots, X_{k} ; \mathbf{P}_{1}, \ldots, \mathbf{P}_{k}\right), \\
& X_{l}=x_{l+1}, \quad \mathbf{P}_{l}=\mathbf{p}_{l+1}, \quad \forall l \in\{1, \ldots, k\}, \quad \text { modulo } k .
\end{aligned}
$$

Remark 5.2. Note that $\pi_{0}=\pi_{12} \pi_{13} \cdots \pi_{1 k}$ and the maps $\pi_{0}, \pi_{i j} \forall i, j \in\{1, \ldots, k\}$, preserve the invariants $H_{1}, H_{2}$ of the Propositions 3.3, 3.9, 3.11. Moreover, the maps $S_{i}:=\pi_{i i+1} R_{i i+1}$, $i \in\{1, \ldots, k\}$, also preserve the invariants $H_{1}, H_{2}$. The following relations holds

$$
S_{i}^{2}=\left(S_{i} S_{i+1}\right)^{3}=\pi_{0}^{k}=\mathrm{id}, \quad\left(S_{i} S_{j}\right)^{2}=\mathrm{id}, \quad|i-j|>1, \quad S_{i} \pi_{0}=\pi_{0} S_{i+1} .
$$

The group $g=\left\langle\pi_{0}, S_{1}, S_{2}, \ldots, S_{k}\right\rangle$ generated by these maps provides a bi-rational realization of the extended Weyl group of type $A_{k-1}^{(1)}$.

Proposition 5.3. The transfer maps $T_{i}^{(k)}$ of a Yang-Baxter map $R$, coincide with the $(k-1)$ iteration of the maps

$$
t_{i}^{(k)}:=\pi_{0} \pi_{i i+1} R_{i i+1}^{\mathbf{p}_{i i+1}}=\pi_{0} S_{i} .
$$

We refer to the maps $t_{i}^{(k)}$ as the extended transfer maps associated with the Yang-Baxter map R. 
Proof. It is enough to show that the $(k-1)$-iteration of the map $t_{1}^{(k)}$ coincides with $T_{1}^{(k)}$. For small values of $k$, this can be proven by direct calculation. In-order to complete the proof, it is enough to show that for arbitrary $k$ the maps $T_{1}^{(k)}$ and $\left(t_{1}^{(k)}\right)^{k-1}$ share the same Lax equation.

Let $L(x, \mathbf{p} ; \boldsymbol{\lambda})$ the Lax matrix associated with the Yang-Baxter map $R$. The Lax equation associated with the transfer map $T_{1}^{(k)}=R_{1 k}^{\mathbf{p}_{1 k}} R_{1 k-1}^{\mathbf{p}_{1 k-1}} \cdots R_{12}^{\mathbf{p}_{12}}$ reads

$$
\begin{aligned}
& L\left(x_{k}, \mathbf{p}_{k} ; \boldsymbol{\lambda}\right) L\left(x_{k-1}, \mathbf{p}_{k-1} ; \boldsymbol{\lambda}\right) \cdots L\left(x_{2}, \mathbf{p}_{2} ; \boldsymbol{\lambda}\right) L\left(x_{1}, \mathbf{p}_{1} ; \boldsymbol{\lambda}\right) \\
& \quad=L\left(X_{1}, \mathbf{p}_{1} ; \boldsymbol{\lambda}\right) L\left(X_{k}, \mathbf{p}_{k} ; \boldsymbol{\lambda}\right) L\left(X_{k-1}, \mathbf{p}_{k-1} ; \boldsymbol{\lambda}\right) \cdots L\left(X_{2}, \mathbf{p}_{2} ; \boldsymbol{\lambda}\right) .
\end{aligned}
$$

Since

$$
\begin{gathered}
\pi_{12} R_{12}^{\mathbf{p}_{12}}: L\left(x_{k}, \mathbf{p}_{k} ; \boldsymbol{\lambda}\right) L\left(x_{k-1}, \mathbf{p}_{k-1} ; \boldsymbol{\lambda}\right) \cdots L\left(x_{2}, \mathbf{p}_{2} ; \boldsymbol{\lambda}\right) L\left(x_{1}, \mathbf{p}_{1} ; \boldsymbol{\lambda}\right) \\
\mapsto L\left(x_{k}, \mathbf{p}_{k} ; \boldsymbol{\lambda}\right) L\left(x_{k-1}, \mathbf{p}_{k-1} ; \boldsymbol{\lambda}\right) \cdots L\left(x_{2}, \mathbf{p}_{2} ; \boldsymbol{\lambda}\right) L\left(x_{1}, \mathbf{p}_{1} ; \boldsymbol{\lambda}\right)
\end{gathered}
$$

and

$$
\begin{aligned}
\pi_{0}: & L\left(x_{k}, \mathbf{p}_{k} ; \boldsymbol{\lambda}\right) L\left(x_{k-1}, \mathbf{p}_{k-1} ; \boldsymbol{\lambda}\right) \cdots L\left(x_{2}, \mathbf{p}_{2} ; \boldsymbol{\lambda}\right) L\left(x_{1}, \mathbf{p}_{1} ; \boldsymbol{\lambda}\right) \\
& \mapsto L\left(x_{1}, \mathbf{p}_{1} ; \boldsymbol{\lambda}\right) L\left(x_{k}, \mathbf{p}_{k} ; \boldsymbol{\lambda}\right) \cdots L\left(x_{3}, \mathbf{p}_{3} ; \boldsymbol{\lambda}\right) L\left(x_{2}, \mathbf{p}_{2} ; \boldsymbol{\lambda}\right)
\end{aligned}
$$

there is

$$
\begin{aligned}
t_{1}^{(k)}: & L\left(x_{k}, \mathbf{p}_{k} ; \boldsymbol{\lambda}\right) L\left(x_{k-1}, \mathbf{p}_{k-1} ; \boldsymbol{\lambda}\right) \cdots L\left(x_{2}, \mathbf{p}_{2} ; \boldsymbol{\lambda}\right) L\left(x_{1}, \mathbf{p}_{1} ; \boldsymbol{\lambda}\right) \\
& \mapsto L\left(x_{1}, \mathbf{p}_{1} ; \boldsymbol{\lambda}\right) L\left(x_{k}, \mathbf{p}_{k} ; \boldsymbol{\lambda}\right) \cdots L\left(x_{3}, \mathbf{p}_{3} ; \boldsymbol{\lambda}\right) L\left(x_{2}, \mathbf{p}_{2} ; \boldsymbol{\lambda}\right)
\end{aligned}
$$

So the map $t_{1}^{(k)}$ has the following Lax equation

$$
\begin{aligned}
& L\left(x_{k}, \mathbf{p}_{k} ; \boldsymbol{\lambda}\right) L\left(x_{k-1}, \mathbf{p}_{k-1} ; \boldsymbol{\lambda}\right) \cdots L\left(x_{2}, \mathbf{p}_{2} ; \boldsymbol{\lambda}\right) L\left(x_{1}, \mathbf{p}_{1} ; \boldsymbol{\lambda}\right) \\
& \quad=L\left(X_{1}, \mathbf{P}_{1} ; \boldsymbol{\lambda}\right) L\left(X_{k}, \mathbf{P}_{k} ; \boldsymbol{\lambda}\right) L\left(X_{k-1}, \mathbf{P}_{k-1} ; \boldsymbol{\lambda}\right) \cdots L\left(X_{2}, \mathbf{P}_{2} ; \boldsymbol{\lambda}\right) .
\end{aligned}
$$

But the map $t_{1}^{(k)}$ acts on the parameter sets $\mathbf{p}_{i}$ as follows

$$
t_{1}^{(k)}:\left(\mathbf{p}_{1}, \ldots, \mathbf{p}_{k}\right) \mapsto\left(\mathbf{P}_{1}, \ldots, \mathbf{P}_{k}\right),
$$

where

$$
\mathbf{P}_{1}=\mathbf{p}_{1}, \quad \mathbf{P}_{k}=\mathbf{p}_{2} \quad \text { and } \quad \forall i \neq 1, k \quad \mathbf{P}_{i}=\mathbf{p}_{i+1},
$$

that is periodic with period $k-1$, so the Lax equation of the map $\left(t_{1}^{(k)}\right)^{k-1}$ is exactly (5.2), i.e., the Lax equation of $T_{1}^{(k)}$.

Theorem 5.4. The maps $t_{i}^{(k)}$ satisfy the relations

$$
\begin{array}{llll}
\left(t_{i}^{(k)} t_{i+1}^{(k)}\right)^{k / 2}=\mathrm{id}, & t_{1}^{(k)} t_{2}^{(k)} \cdots t_{k}^{(k)}=\mathrm{id}, & k & \text { even } \\
\left(t_{i}^{(k)} t_{i+i}^{(k)}\right)^{k}=\mathrm{id}, & \left(t_{1}^{(k)} t_{2}^{(k)} \cdots t_{k}^{(k)}\right)^{2}=\mathrm{id}, \quad & k & \text { odd } .
\end{array}
$$

Proof. Let us first prove that $t_{1}^{(k)} t_{2}^{(k)} \cdots t_{k}^{(k)}=\mathrm{id}$ for $k=2 m$ even. There is

$$
t_{1}^{(2 m)} t_{2}^{(2 m)} \cdots t_{2 m}^{(2 m)}=\pi_{0} S_{1} \pi_{0} S_{2} \cdots \pi_{0} S_{2 m},
$$


where we have the composition of $m$ expressions of the form $\pi_{0} S_{i} \pi_{0} S_{i+1}$, and for each one of them (using Remark 5.2) it holds $\pi_{0} S_{i} \pi_{0} S_{i+1}=\pi_{0} S_{i}^{2} \pi_{0}=\pi_{0}^{2}$. So

$$
t_{1}^{(2 m)} t_{2}^{(2 m)} \cdots t_{2 m}^{(2 m)}=\underbrace{\pi_{0}^{2} \pi_{0}^{2} \cdots \pi_{0}^{2}}_{m \text {-times }}=\pi_{0}^{2 m}=\mathrm{id} .
$$

Let us now prove that $\left(t_{i}^{(k)} t_{i+1}^{(k)}\right)^{k / 2}=\mathrm{id}$. We have

$$
\left(t_{i}^{(k)} t_{i+1}^{(k)}\right)^{k / 2}=\left(t_{i}^{(k)} t_{i+1}^{(k)}\right)^{m}=\left(\pi_{0} S_{i} \pi_{0} S_{i+1}\right)^{m}=\left(\pi_{0}^{2} S_{i+1}^{2}\right)^{m}=\pi_{0}^{2 m}=\mathrm{id} .
$$

For $k=2 m+1$ odd, we have

$$
\left(t_{i}^{(k)} t_{i+1}^{(k)}\right)^{k}=\left(t_{i}^{(k)} t_{i+1}^{(k)}\right)^{2 m+1}=\left(\pi_{0}^{2} S_{i+1}^{2}\right)^{2 m+1}=\left(\pi_{0}^{2 m+1}\right)^{2}=\mathrm{id} .
$$

Also,

$$
\begin{aligned}
\left(t_{1}^{(2 m+1)} t_{2}^{(2 m+1)} \cdots t_{2 m+1}^{(2 m+1)}\right)^{2} & =\left(t_{1}^{(2 m+1)} t_{2}^{(2 m+1)} \cdots t_{2 m}^{(2 m+1)} \pi_{0} S_{2 m+1}\right)^{2} \\
& =\left(\pi_{0}^{2 m+1} S_{2 m+1}\right)^{2}=S_{2 m+1}^{2}=\mathrm{id}
\end{aligned}
$$

where we have used the fact that

$$
t_{1}^{(2 m+1)} t_{2}^{(2 m+1)} \cdots t_{2 m}^{(2 m+1)}=\underbrace{\pi_{0}^{2} \pi_{0}^{2} \cdots \pi_{0}^{2}}_{m \text {-times }}=\pi_{0}^{2 m} .
$$

Remark 5.5. Note that for $k$ odd, it holds the more general condition

$$
\left(t_{i}^{(k)} t_{j}^{(k)}\right)^{k}=\mathrm{id}, \quad i \neq j .
$$

\section{$5.2 k$-point recurrences associated with the transfer maps of the $\boldsymbol{H}$-list of quadrirational Yang-Baxter maps}

We refer to the extended transfer maps $t_{i}^{(k)}$ that correspond to the $H_{\mathrm{I}}, H_{\mathrm{II}}, H_{\mathrm{III}}^{A}, H_{\mathrm{III}}^{B}$ and $H_{\mathrm{V}}$ Yang-Baxter maps respectively as $t_{i}^{H_{\mathrm{I}}(k)}, t_{i}^{H_{\mathrm{II}}(k)}, t_{i}^{H_{\mathrm{III}}^{A}(k)}, t_{i}^{H_{\mathrm{III}}^{B}(k)}$ and $t_{i}^{H_{\mathrm{V}}(k)}$.

Here, we associate $k$-point recurrences with the maps $t_{i}^{H_{\mathrm{I}}(k)}, t_{i}^{H_{\mathrm{II}}(k)}, t_{i}^{H_{\mathrm{III}}^{A}(k)}, t_{i}^{H_{\mathrm{III}}^{B}(k)}$ and $t_{i}^{H_{\mathrm{V}}(k)}$. Let us first introduce the shift operator $T$ as follows

$$
\begin{aligned}
& T^{0}: x(n) \mapsto x(n), \quad T^{1}: x(n) \mapsto x(n+1), \quad T^{l}: x(n) \mapsto x(n+l), \\
& T^{-l}: x(n) \mapsto x(n-l), \quad n, l \in \mathbb{Z} .
\end{aligned}
$$

The maps $t_{2}^{H_{\mathrm{I}}(k)}, t_{2}^{H_{\mathrm{II}}(k)}, t_{2}^{H_{\mathrm{III}}^{A}(k)}, t_{2}^{H_{\mathrm{III}}^{B}(k)}$ and $t_{2}^{H_{\mathrm{V}}(k)}$, explicitly read

$$
\left(x_{1}, \ldots, x_{k} ; p_{1}, \ldots, p_{k}\right) \mapsto\left(T x_{1}, \ldots, T x_{k} ; T p_{1}, \ldots, T p_{k}\right),
$$

where

$$
\begin{aligned}
& T x_{1}=x_{2} \frac{p_{3}\left(1-p_{2}\right)+\left(p_{2}-p_{3}\right) x_{3}+\left(p_{3}-1\right) x_{2} x_{3}}{p_{2}\left(1-p_{3}\right)+\left(p_{3}-p_{2}\right) x_{2}+\left(p_{2}-1\right) x_{2} x_{3}}, \quad T p_{1}=p_{3}, \quad T x_{i}=x_{i+1}, \\
& T x_{2}=x_{3} \frac{p_{2}\left(1-p_{3}\right)+\left(p_{3}-p_{2}\right) x_{2}+\left(p_{2}-1\right) x_{2} x_{3}}{p_{3}\left(1-p_{2}\right)+\left(p_{2}-p_{3}\right) x_{3}+\left(p_{3}-1\right) x_{2} x_{3}}, \quad T p_{2}=p_{2}, \quad T p_{i}=p_{i+1}, \quad\left(t_{2}^{H_{\mathrm{I}}(k)}\right) \\
& T x_{1}=p_{3} x_{2} \frac{x_{2}+x_{3}-p_{2}}{p_{3} x_{2}+p_{2} x_{3}-p_{2} p_{3}}, \quad T p_{1}=p_{3}, \quad T x_{i}=x_{i+1},
\end{aligned}
$$




$$
\begin{array}{llll}
T x_{2}=p_{2} x_{3} \frac{x_{2}+x_{3}-p_{3}}{p_{3} x_{2}+p_{2} x_{3}-p_{2} p_{3}}, \quad T p_{2}=p_{2}, \quad T p_{i}=p_{i+1}, & \left(t_{2}^{H_{\mathrm{II}}(k)}\right) \\
T x_{1}=\frac{x_{2}}{p_{3}} \frac{p_{2} x_{2}+p_{3} x_{3}}{x_{2}+x_{3}}, \quad T p_{1}=p_{3}, & T x_{i}=x_{i+1}, & \\
T x_{2}=\frac{x_{3}}{p_{2}} \frac{p_{2} x_{2}+p_{3} x_{3}}{x_{2}+x_{3}}, & T p_{2}=p_{2}, & T p_{i}=p_{i+1}, & \left(t_{2}^{H_{\mathrm{III}}^{A}(k)}\right) \\
T x_{1}=x_{2} \frac{1+p_{2} x_{2} x_{3}}{1+p_{3} x_{2} x_{3}}, & T p_{1}=p_{3}, & T x_{i}=x_{i+1}, & \\
T x_{2}=x_{3} \frac{1+p_{3} x_{2} x_{3}}{1+p_{2} x_{2} x_{3}}, & T p_{2}=p_{2}, & T p_{i}=p_{i+1}, & \\
T x_{1}=x_{2}-\frac{p_{3}-p_{2}}{x_{2}+x_{3}}, & T p_{1}=p_{3}, & T x_{i}=x_{i+1}, & \\
T x_{2}=x_{3}+\frac{p_{3}-p_{2}}{x_{2}+x_{3}}, & T p_{2}=p_{2}, & T p_{i}=p_{i+1}, & \left(t_{2}^{H_{\mathrm{V}}(k)}\right)
\end{array}
$$

with $i=3,4, \ldots, k$ and $T x_{k}=x_{1}, T p_{k}=p_{1}$. Moreover, not just $t_{2}^{(k)}$, but all the maps $t_{i}^{(k)}, i=$ $1,2, \ldots, k$, preserve the invariants in separated variables (see Table 10$)^{4}$ and they anti-preserve the measures $m_{i}=n^{i} d^{i+1}$ where $n^{i}, d^{i}$ the numerator and the denominator respectively of the invariants $H_{i}, i=1,2$. Additional invariant can be constructed though the Lax formulation (see the proof of Proposition 5.3).

Table 10. Invariants in separated variables for the maps $t_{i}^{H_{\mathrm{I}}(k)}, t_{i}^{H_{\mathrm{II}}(k)}, t_{i}^{H_{\mathrm{III}}^{A}(k)}, t_{i}^{H_{\mathrm{III}}^{B}(k)}$ and $t_{i}^{H_{\mathrm{V}}(k)}$.

\begin{tabular}{c|c|c}
\hline map & $H_{1}$ & $H_{2}$ \\
\hline$t_{i}^{H_{\mathrm{I}}(k)}$ & $\prod_{i=1}^{k} p_{i} x_{i}$ & $\prod_{i=1}^{k} \frac{x_{i}-p_{i}}{x_{i}-1} \frac{1}{p_{i}-1}$ \\
$t_{i}^{H_{\mathrm{II}}(k)}$ & $\sum_{i=1}^{k} x_{i}+p_{i}$ & $\prod_{i=1}^{k} \frac{x_{i}-p_{i}}{p_{i} x_{i}}$ \\
$t_{i}^{H_{\mathrm{III}}^{A}(k)}$ & $\sum_{i=1}^{k} \frac{1}{x_{i}}+\frac{1}{p_{i}}$ & $\sum_{i=1}^{k} p_{i} x_{i}$ \\
$t_{i}^{H_{\mathrm{III}}^{B}(k)}$ & $\prod_{i=1}^{k} p_{i} x_{i}$ & $\sum_{i=1}^{k} \frac{1}{x_{i}}+p_{i} x_{i}+\frac{1}{p_{i}}$ \\
$t_{i}^{H_{\mathrm{V}}(k)}$ & $\sum_{i=1}^{k} x_{i}+p_{i}$ & $\sum_{i=1}^{k} x_{i}^{3}+3 p_{i} x_{i}+p_{i}^{3}$ \\
\hline
\end{tabular}

Now we show how a $k$-point recurrence can be associated with the map $t_{2}^{H_{\mathrm{V}}(k)}$. Recall that the map $t_{2}^{H_{\mathrm{V}}(k)}$ reads

$$
t_{2}^{H_{\mathrm{V}}(k)}:\left(x_{1}, \ldots, x_{k} ; p_{1}, \ldots, p_{k}\right) \mapsto\left(T x_{1}, \ldots, T x_{k} ; T p_{1}, \ldots, T p_{k}\right)
$$

where

$$
\begin{aligned}
& T x_{1}=x_{2}-\frac{p_{3}-p_{2}}{x_{2}+x_{3}}, \quad T x_{2}=x_{3}+\frac{p_{3}-p_{2}}{x_{2}+x_{3}}, \quad T x_{i}=x_{i+1}, \\
& T p_{1}=p_{3}, \quad T p_{2}=p_{2}, \quad T p_{i}=p_{i+1}, \quad i=3, \ldots, k,
\end{aligned}
$$

\footnotetext{
${ }^{4}$ The invariants in separated variables that appear in Table 10, were firstly introduced, in a different context, in $[44,45,47,56]$. Note that the invariants $H_{1}, H_{2}$ for $t_{i}^{H_{\mathrm{III}}^{A}(k)}$ were also given in [50].
} 
and the indices are considered modulo $k$. Clearly we have, $x_{3}=T^{2-k} x_{1}, p_{3}=T^{2-k} p_{1}$. So we obtain

$$
\begin{aligned}
& T x_{1}=x_{2}-\frac{T^{2-k} p_{1}-p_{2}}{x_{2}+T^{2-k} x_{1}}, \quad T x_{2}=T^{2-k} x_{1}+\frac{T^{2-k} p_{1}-p_{2}}{x_{2}+T^{2-k} x_{1}}, \\
& T^{k-1} p_{1}=p_{1}, \quad T p_{2}=p_{2} .
\end{aligned}
$$

Adding the first two equations from above we get the following invariance condition ${ }^{5}$

$$
\left(T^{1}-T^{2-k}\right) x_{1}=\left(T^{0}-T^{1}\right) x_{2} .
$$

So it is guaranteed the existence of a potential function $f$ such that

$$
x_{1}=c+\left(T^{0}-T^{1}\right) f, \quad x_{2}=c+\left(T^{1}-T^{2-k}\right) f, \quad \text { where } \quad c=\text { const. }
$$

In terms of $f,(5.3)$ becomes the following $(k+1)$-point recurrence

$$
\left(T^{2}-T^{2-k}\right) f=\frac{-p_{2}+T^{2-k} p_{1}}{2 c+\left(T-T^{3-k}\right) f}, \quad T^{k-1} p_{1}=p_{1}, \quad T p_{2}=p_{2} .
$$

In terms of a new variable $h$ defined as $h:=\lambda+\left(T^{1}-T^{0}\right) f$, there is,

$$
\left(T^{2}-T^{2-k}\right) f=-\lambda k+\sum_{i=2-k}^{1} T^{i} h, \quad\left(T-T^{3-k}\right) f=\lambda(2-k)+\sum_{i=3-k}^{0} T^{i} h,
$$

so (5.5) becomes the $k$-point recurrence

$$
\frac{2 c k}{2-k}+\sum_{i=2-k}^{1} T^{i} h=\frac{-p_{2}+T^{2-k} p_{1}}{\sum_{i=3-k}^{0} T^{i} h}, \quad T^{k-1} p_{1}=p_{1}, \quad T p_{2}=p_{2},
$$

where we chose $\lambda=\frac{2 c}{k-2}$ to simplify the formulae.

Table 11. The invariance conditions (5.4) and the potential functions $f$ for the maps $t_{2}^{H_{\mathrm{I}}(k)}, t_{2}^{H_{\mathrm{II}}(k)}$, $t_{2}^{H_{\mathrm{III}}^{A}(k)}, t_{2}^{H_{\mathrm{III}}^{B}(k)}$ and $t_{2}^{H_{\mathrm{V}}(k)}$.

\begin{tabular}{c|c|c}
\hline map & invariance condition & potential function $f$ \\
\hline$t_{2}^{H_{\mathrm{I}}(k)}$ & $\frac{T x_{1}}{T^{2-k} x_{1}}=\frac{T^{0} x_{2}}{T x_{2}}$ & $x_{1}=c \frac{T^{0} f}{T f}, x_{2}=c \frac{T f}{T^{2-k} f}$ \\
$t_{2}^{H_{\mathrm{II}}(k)}$ & $\left(T-T^{2-k}\right) x_{1}=\left(T^{0}-T\right) x_{2}$ & $x_{1}=c+\left(T^{0}-T\right) f, x_{2}=c+\left(T-T^{2-k}\right) f$ \\
$t_{2}^{H_{\text {III }}^{A}(k)}$ & $\left(T-T^{2-k}\right) \frac{1}{x_{1}}=\left(T^{0}-T\right) \frac{1}{x_{2}}$ & $\frac{1}{x_{1}}=\frac{1}{c}+\left(T^{0}-T\right) f, \frac{1}{x_{2}}=\frac{1}{c}+\left(T-T^{2-k}\right) f$ \\
$t_{2}^{H_{\text {III }}^{B}(k)}$ & $\frac{T x_{1}}{T^{2-k} x_{1}}=\frac{T^{0} x_{2}}{T x_{2}}$ & $x_{1}=c \frac{T^{0} f}{T f}, x_{2}=c \frac{T f}{T^{2-k} f}$ \\
$t_{2}^{H_{\mathrm{V}}(k)}$ & $\left(T-T^{2-k}\right) x_{1}=\left(T^{0}-T\right) x_{2}$ & $x_{1}=c+\left(T^{0}-T\right) f, x_{2}=c+\left(T-T^{2-k}\right) f$ \\
\hline
\end{tabular}

${ }^{5}$ This condition is a consequence of the fact that the $t_{i}^{H_{\mathrm{V}}(k)}$ preserves the invariant $H_{1}=\sum_{i=1}^{k} x_{i}$. Such a condition exists for the remaining extended transfer maps associated with the Yang-Baxter maps of the $H$-list. The latter enable us to write $t_{2}^{(k)}$ maps as $k$-point recurrences. 
Proposition 5.6. The following $(k+1)$-point recurrences corresponds to the extended transfer map $t_{2}^{(k)}$ associated with $H_{\mathrm{I}}, H_{\mathrm{II}}, H_{\mathrm{III}}^{A}, H_{\mathrm{III}}^{B}$ and $H_{\mathrm{V}}$ Yang-Baxter maps respectively. We refer to these $(k+1)$-point recurrences respectively as $r t_{2}^{H_{\mathrm{I}}(k)}, r t_{2}^{H_{\mathrm{I}}(k)}, r t_{2}^{H_{\mathrm{III}}^{A}(k)}, r t_{2}^{H_{\mathrm{III}}^{B}(k)}$ and $r t_{2}^{H_{\mathrm{V}}(k)}$

$$
\begin{aligned}
& \frac{T^{2} f}{T^{2-k} f}=\frac{p_{2}\left(-1+T^{2-k} p_{1}\right)+c\left(p_{2}-T^{2-k} p_{1}\right) \frac{T^{1} f}{T^{2-k} f}+c^{2}\left(1-p_{2}\right) \frac{T^{1} f}{T^{3-k} f}}{\left(T^{2-k} p_{1}\right)\left(p_{2}-1\right)+c\left(-p_{2}+T^{2-k} p_{1}\right) \frac{T^{2-k} f}{T^{3-k} f}+c^{2}\left(1-T^{2-k} p_{1}\right) \frac{T^{1} f}{T^{3-k} f}},\left(r t_{2}^{H_{\mathrm{I}}(k)}\right) \\
& \frac{c+\left(T^{1}-T^{2}\right) f}{c+\left(T^{1}-T^{2-k}\right) f} \\
& =\frac{\left(2 c-p_{2}+\left(T^{1}-T^{3-k}\right) f\right) T^{2-k} p_{1}}{-p_{2} T^{2-k} p_{1}+c\left(p_{2}+T^{2-k} p_{1}\right)+\left(T^{2-k} p_{1}\right)\left(T^{1}-T^{2-k}\right) f+p_{2}\left(T^{2-k}-T^{3-k}\right) f},\left(r t_{2}^{H_{\text {II }}(k)}\right) \\
& \frac{c+\left(T^{1}-T^{2}\right) f}{c+\left(T^{1}-T^{2-k}\right) f}=\frac{2 c+\left(T^{1}-T^{3-k}\right) f}{c+\left(T^{1}-T^{2-k}\right) f+\frac{p_{2}}{T^{2-k} p_{1}}\left(c+\left(T^{2-k}-T^{3-k}\right) f\right)}, \quad\left(r t_{2}^{H_{\mathrm{III}}^{A}(k)}\right) \\
& \frac{T^{2} f}{T^{2-k} f}=\frac{T^{3-k} f+c^{2}\left(T^{2-k} p_{1}\right) T^{1} f}{T^{3-k} f+c^{2} p_{2} T^{1} f}, \quad \quad\left(r t_{2}^{H_{\mathrm{III}}^{B}(k)}\right) \\
& \left(T^{2}-T^{2-k}\right) f=\frac{-p_{2}+T^{2-k} p_{1}}{2 c+\left(T-T^{3-k} f\right)} . \quad \quad\left(r t_{2}^{H_{\mathrm{V}}(k)}\right)
\end{aligned}
$$

For each recurrence presented above we have that the parameters vary as follows: $T p_{2}=p_{2}$, $T^{k-1} p_{1}=p_{1}$. So $p_{2}$ is constant and $p_{1}$ is periodic with period $k-1$.

Note that the recurrences $r t_{2}^{H_{\mathrm{I}}(k)}$ and $r t_{2}^{H_{\mathrm{III}}^{B}(k)}$ are bilinear. Some members of $r t_{2}^{H_{\mathrm{I}}(k)}$ and $r t_{2}^{H_{\mathrm{III}}^{B}(k)}$, for specific choices of the parameters $c, p_{2}$ and of the function $p_{1}$, are expected to exhibit the Laurent property [26, 27, 28].

Table 12. Definition of the variables $h$ associated with the recurrences of Proposition 5.6.

\begin{tabular}{c|c|c}
\hline recurrence & variable $h$ & a choice for $\lambda$ \\
\hline$r t_{2}^{H_{\mathrm{I}}(k)}$ & $h:=\lambda \frac{T f}{T^{0} f}$ & $\lambda=\frac{1}{c}$ \\
$r t_{2}^{H_{\mathrm{II}}(k)}$ & $h:=\lambda+\left(T-T^{0}\right) f$ & $\lambda=\frac{2 c}{k-2}$ \\
$r t_{2}^{H_{\mathrm{III}}^{A}(k)}$ & $h:=\lambda+\left(T-T^{0}\right) f$ & $\lambda=\frac{2 c}{k-2}$ \\
$r t_{2}^{H_{\mathrm{III}}^{B}(k)}$ & $h:=\lambda \frac{T f}{T^{0} f}$ & $\lambda=\frac{1}{c}$ \\
$r t_{2}^{H_{\mathrm{V}}(k)}$ & $h:=\lambda+\left(T-T^{0}\right) f$ & $\lambda=\frac{2 c}{k-2}$ \\
\hline
\end{tabular}

Corollary 5.7. The $(k+1)$-point recurrences $r t_{2}^{H_{\mathrm{I}}(k)}, r t_{2}^{H_{\mathrm{II}}(k)}, r t_{2}^{H_{\mathrm{III}}^{A}(k)}, r t_{2}^{H_{\mathrm{III}}^{B}(k)}$ and $r t_{2}^{H_{\mathrm{V}}(k)}$, in terms of the corresponding variables $h$ defined in Table 12, get the form of the following $k$-point recurrences

$$
\prod_{i=3-k}^{1} T^{i} h=\frac{c^{-k} p_{2}\left(T^{2-k} p_{1}-1\right)+\left(p_{2}-T^{2-k} p_{1}\right) \prod_{i=2-k}^{0} T^{i} h+\left(1-p_{2}\right) \prod_{i=3-k}^{0} T^{i} h}{T^{2-k} p_{1}-p_{2}+T^{2-k} p_{1}\left(p_{2}-1\right) T^{2-k} h+c^{k}\left(1-T^{2-k} p_{1}\right) \prod_{i=3-k}^{0} T^{i} h},\left(\hat{r} t_{2}^{H_{\mathrm{I}}(k)}\right)
$$




$$
\begin{array}{cc}
p_{2}\left(\frac{c k}{k-2}-T h\right)\left(\frac{c k}{k-2}-T^{2-k} p_{1}-T^{2-k} h\right) & \\
=T^{2-k} p_{1}\left(\frac{c k}{k-2}-\sum_{i=2-k}^{0} T^{i} h\right)\left(\frac{c k}{k-2}+p_{2}-\sum_{i=3-k}^{1} T^{i} h\right), & \left(\hat{r} t_{2}^{H_{\mathrm{II}}(k)}\right) \\
p_{2}\left(\frac{c k}{k-2}-T h\right)\left(\frac{c k}{k-2}-T^{2-k} h\right) & \\
=T^{2-k} p_{1}\left(\frac{c k}{k-2}-\sum_{i=2-k}^{0} T^{i} h\right)\left(\frac{c k}{k-2}-\sum_{i=3-k}^{1} T^{i} h\right), & \left(\hat{r} t_{2}^{H_{\mathrm{III}}^{A}(k)}\right) \\
\prod_{i=2-k}^{1} T^{i} h=\frac{c^{-k}+T^{2-k} p_{1} \prod_{i=3-k}^{0} T^{i} h}{\prod_{i=3-k}^{0} T^{i} h} & \left(\hat{r} t_{2}^{H_{\mathrm{III}}^{B}(k)}\right) \\
-\frac{1+c^{k} p_{2}}{k-2}+\sum_{i=2-k}^{1} T^{i} h=\frac{-p_{2}+T^{2-k} p_{1}}{0} & \\
\sum_{i=3-k}^{0} T^{i} h & \left(\hat{r} t_{2}^{H_{\mathrm{V}}(k)}\right) \\
\end{array}
$$

and for each recurrence presented above we have that the parameters vary as follows: $T p_{2}=p_{2}$, $T^{k-1} p_{1}=p_{1}$. So $p_{2}$ is constant and $p_{1}$ is periodic with period $k-1$.

Note that the $(k+1)$-point recurrences of Proposition 5.6, as well as the corresponding $k$-point ones introduced in Corollary 5.7 are non-autonomous. This is due to the fact that $p_{1}$ varies periodically $\left(T^{k-1} p_{1}=p_{1}\right)$. The non-autonomous terms that will be introduced by integrating the relation $T^{k-1} p_{1}=p_{1}$ are periodic though. Proper de-autonomization for the recurrences $\hat{r} t_{2}^{H_{\mathrm{V}}(k)}$ and $\hat{r} t_{2}^{H_{\mathrm{III}}^{B}(k)}$ will be introduced in what follows.

\subsubsection{The recurrences $\hat{r} t_{i}^{H_{\mathrm{V}}(k)}$ and discrete Painlevé equations}

The dressing chain for the KdV equation [71], reads

$$
\left(g_{i+1}+g_{i}\right)_{t}=g_{i+1}^{2}-g_{i}^{2}+p_{i+1}-p_{i} .
$$

The recurrences $\hat{r} t_{i}^{H_{\mathrm{V}}(k)}$, serve as its discretisations. Actually they are exactly the $(k-1)$-roots of the discretisations presented in [1]. So, $\hat{r} t_{i}^{H_{\mathrm{V}}(k)}$ corresponds to Liouville integrable maps.

Since the dressing chain (5.6) leads to Painlevé equations $P_{\mathrm{IV}}$ and $P_{\mathrm{V}}$ and their higher order analogues [71], the recurrences $\hat{r} t_{i}^{H_{\mathrm{V}}{ }^{(k)}}$ (after proper de-autonomisation) can be considered as their discrete counter-parts and/or the Bäcklund transformations of the higher order $P_{\mathrm{IV}}$ and $P_{\mathrm{V}}$ Painlevé equations.

A proper de-autonomisation of $\hat{r} t_{2}^{H_{\mathrm{V}}(k)}$ is achieved by breaking the periodicity of the $p_{1}$ assuming that $T^{k-1} p_{1}=p_{1}+(k-1) a$, where $a$ constant. This de-autonomisation is proper since the resulting non-autonomous discrete system preserves the same Poisson structure ${ }^{6}$ as the autonomous one. So we obtain the following hierarchy of discrete Painlevé equations

$$
-\frac{2 c k}{k-2}+\sum_{i=2-k}^{1} T^{i} h=\frac{-p_{2}+T^{2-k} p_{1}}{\sum_{i=3-k}^{0} T^{i} h}, \quad T p_{2}=p_{2}, \quad T^{k-1} p_{1}=p_{1}+(k-1) a .
$$

\footnotetext{
${ }^{6}$ The Poisson structures associated with the dressing chain for the KdV equation were first derived in [71], see also [25].
} 
For $k=3,(5.7)$ reads

$$
-6 c+T h+h+T^{-1} h=\frac{-p_{2}+T^{-1} p_{1}}{h}, \quad T p_{2}=p_{2}, \quad T^{2} p_{1}=p_{1}+2 a .
$$

So $p_{2}$ is constant and $p_{1}=b_{0}+b_{1}(-1)^{n}+a n$, with $b_{0}, b_{1}$, a constants. We can choose $-p_{2}+b_{0}=b$ constant, hence we obtain the following discrete Painlevé equation which serves as Bäcklund transformation of $P_{\mathrm{IV}}[57]$

$$
-6 c+T h+h+T^{-1} h=\frac{b+b_{1}(-1)^{n}+a n}{h}, \quad n \in \mathbb{Z} .
$$

For $k=4,(5.7)$ reads

$$
-4 c+T^{-2} h+h+T^{-1} h+h+T h=\frac{-p_{2}+T^{-2} p_{1}}{h+T^{-1} h}, \quad T p_{2}=p_{2}, \quad T^{3} p_{1}=p_{1}+3 a .
$$

If we define a new variable $w$ as $w:=h+T^{-1} h$, then we obtain the following discrete Painlevé equation which serves as Bäcklund transformation of $P_{\mathrm{V}}$

$$
-4 c+T^{-1} w+T w=\frac{-p_{2}+T^{-2} p_{1}}{w}, \quad T p_{2}=p_{2}, \quad T^{3} p_{1}=p_{1}+3 a .
$$

So for $k$ odd (5.7) serves as Bäcklund transformation for the higher order analogues of $P_{\mathrm{IV}}$ and for $k$ even (5.7) serves as Bäcklund transformation for the higher order analogues of $P_{\mathrm{V}}$. Note that in [57], Bäcklund transformation for the higher order analogues of $P_{\mathrm{IV}}$ and $P_{\mathrm{V}}$ were given in terms of continued fractions. We can recover the form of discrete Painlevé equations introduced in [57] by making use of the alternating terms that appear in (5.7). For example for $k=3$, the term $(1)^{n}$ that appears in (5.8), suggests the introduction of the variables $y(m):=h(2 n)$, $z(m):=h(2 n+1)$. Then $(5.8)$ takes to form of the second discrete Painlevé equation $d P_{\mathrm{II}}$

$$
y+z+T^{-1} z=\frac{b_{0}+b_{1}+a m}{y}, \quad T y+y+z=\frac{b_{0}-b_{1}+a m}{z}, \quad m \in \mathbb{Z} .
$$

\subsubsection{The recurrences $\hat{r} t_{i}^{H_{\mathrm{III}}^{B}(k)}$ and discrete Painlevé equations}

As we plan to show in our future work, the recurrences $\hat{r} t_{i}^{H_{\mathrm{III}}^{B}(k)}$ serves as Liouville integrable discretisations of the following chain introduced in [6]

$$
\left(g_{i}+g_{i+1}\right)_{t}=2\left(p_{i} \cosh g_{i}-p_{i+1} \cosh g_{i+1}\right) .
$$

A proper de-autonomisation of $\hat{r} t_{2}^{H_{\mathrm{III}}^{B}(k)}$ is achieved by breaking the periodicity of the $p_{1}$ in a way that the non-autonomous system preserves the same Poisson structure as the autonomous one. This is achieved by imposing that $T^{k-1} p_{1}=p_{1} a^{k-1}$, where $a$ constant. So we obtain the following hierarchy of discrete Painlevé equations

$$
\prod_{i=2-k}^{1} T^{i} h=\frac{c^{-k}+T^{2-k} p_{1} \prod_{i=3-k}^{0} T^{i} h}{1+c^{k} p_{2} \prod_{i=3-k}^{0} T^{i} h}, \quad T p_{2}=p_{2}, \quad T^{k-1} p_{1}=p_{1} a^{k-1}
$$

For $k=3,(5.9)$ reads

$$
T h T^{-1} h=\frac{1}{h} \frac{c^{-3}+h T^{-1} p_{1}}{1+c^{3} p_{2} h}, \quad T p_{2}=p_{2}, \quad T^{2} p_{1}=p_{1} a^{2} .
$$


So $p_{2}$ is constant and $p_{1}=b_{0} a^{n}+b_{1}(-a)^{n}$, with $b_{0}, b_{1}, a$ constants. Hence we obtain the $q-P_{\mathrm{I}}\left(A_{6}^{(1)}\right)$ discrete Painlevé equation (see [63]). For $k=4,(5.9)$ reads

$$
T h T^{0} h T^{-1} h T^{-2} h=\frac{c^{-4}+h T^{-1} h T^{-2} p_{1}}{1+c^{4} p_{2} h T^{-1} h}, \quad T p_{2}=p_{2}, \quad T^{3} p_{1}=p_{1} a^{3} .
$$

If we define a new variable $w$ as $w:=h T^{-1} h$, then we obtain the $q-P_{\mathrm{II}}\left(A_{5}^{(1)}\right)$ discrete Painlevé equation (see [63])

$$
T w T^{-1} w=\frac{c^{-4}+w T^{-2} p_{1}}{1+c^{4} p_{2} w}, \quad T p_{2}=p_{2}, \quad T^{3} p_{1}=p_{1} a^{3} .
$$

The Lax pair associated with the hierarchy (5.9) first appeared in [32].

Remark 5.8. As for the recurrences $\hat{r} t_{i}^{H_{\mathrm{III}}^{A}(k)}, \hat{r} t_{i}^{H_{\mathrm{II}}(k)}$, one could consider $T^{k-1} p_{1}=p_{1}+(k-1) a$ and for $\hat{r} t_{i}^{H_{\mathrm{I}}(k)} T^{k-1} p_{1}=p_{1} a^{k-1}$, in order to de-autonomise them. We anticipate that this is a proper de-autonomisation, although we have no proof yet. The finding of the Poisson structures that the latter recurrences we anticipate that preserve, will sort this issue out.

Remark 5.9. As a final remark, we note that the $k$-point recurrences associated with the extended transfer maps of the Yang-Baxter map $F_{\mathrm{V}}$, are exactly the same as the $k$-point recurrences associated with the extended transfer maps of the Yang-Baxter map $H_{\mathrm{V}}$ which (one of them) were presented in Corollary 5.7. Since the $(k-1)$-iteration of the extended transfer maps of any Yang-Baxter map coincides with its transfer maps, we conclude that the dynamics of the transfer maps of the Yang-Baxter maps $F_{\mathrm{V}}$ and $H_{\mathrm{V}}$, are the same. The same holds true for the transfer maps associated with the Yang-Baxter maps $F_{\mathrm{III}}$ and $H_{\mathrm{III}}^{A}$. As for the remaining members of the $F$ and the $H$ lists of Yang-Baxter maps, further investigation is required in order to prove the equivalence of their transfer dynamics.

\section{Conclusions}

In Section 2 we have presented a family of maps in $k$ variables which preserve 2 rational invariants of a specific form. One could mimic the procedures introduced in [29] to obtain rational maps in $k$ variables which preserve $m$ rational invariants where $m<k$. For example, there are $\left(\begin{array}{c}2 k \\ k\end{array}\right)$ rational maps $\left(x_{1}, \ldots, x_{k}, y_{1}, \ldots, y_{k}\right) \mapsto\left(X_{1}, \ldots, X_{k}, Y_{1}, \ldots, Y_{k}\right)$ which preserve $k$ invariants of the form:

$$
H_{i}=\frac{\alpha_{i} x_{i} x_{i+1}+\beta_{i} x_{i}+\gamma_{i} x_{i+1}+\delta_{i}}{\kappa_{i} x_{i} x_{i+1}+\lambda_{i} x_{i}+\mu_{i} x_{i+1}+\nu_{i}}, \quad i=1,2, \ldots, k,
$$

where the indices are considered modulo $k$ and $\alpha_{i}, \beta_{i}, \kappa_{i}, \lambda_{i}$, etc. are given functions of the variables $y_{i}, y_{i+1}$.

If separability of variables on the invariants is imposed, then higher rank analogues of the Yang-Baxter maps of Propositions 3.3, 3.9 and 3.11 are expected. Moreover, solutions of the functional tetrahedron equation [41, 42, 49, 64], or even of higher simplex equations [17, 53, 54] are anticipated. For example if we consider the following, different than (6.1), choice of invariants:

$$
H_{1}=\sum_{i=1}^{6} x_{i}, \quad H_{2}=\frac{x_{1} x_{4} x_{6}}{x_{3}}, \quad H_{3}=x_{2} x_{3} x_{4} x_{5},
$$


then the involutions $R_{123}, R_{145}, R_{246}$, and $R_{356}$, preserve $H_{i}, i=1,2,3$ and satisfy the functional tetrahedron equation

$$
R_{123} R_{145} R_{246} R_{356}=R_{356} R_{246} R_{145} R_{123} .
$$

They are exactly the Hirota's map [41, 42, 64], i.e., the map $R:(u, v, w) \mapsto(U, V, W)$, where

$$
U=\frac{u v}{u+w}, \quad V=u+w, \quad W=\frac{v w}{u+w},
$$

acting on (123), (145), (246) and (356) coordinates respectively. For the involution $\phi: u \mapsto-u$, it holds $\phi_{1} \phi_{2} \phi_{3} R_{123}=R_{123} \phi_{1} \phi_{2} \phi_{3}$. So $\phi$ is a symmetry of the Hirota's map $R$ and it can be easily proven that the following entwining relation holds

$$
R_{123} \phi_{3} R_{145} \phi_{5} R_{246} \phi_{6} R_{356}=R_{356} R_{246} \phi_{6} R_{145} \phi_{5} R_{123} \phi_{3} .
$$

Hence we have obtained a solution of the following entwining functional tetrahedron relation

$$
S_{123} S_{145} S_{246} T_{356}=T_{356} S_{246} S_{145} S_{123},
$$

where $T$ is the Hirota's map acting on the (356) coordinates and $S:(u, v, w) \mapsto(U, V, W)$ a non-periodic map where

$$
U=\frac{u v}{u-w}, \quad V=u-w, \quad W=-\frac{v w}{u-w} .
$$

The complete set of entwining relations and maps associated with the Hirota's map as well as with the Hirota-Miwa's map, will be considered elsewhere.

In Section 4, we considered two methods to obtain entwining maps. The first method uses degeneracy arguments and produces entwining maps associated with the $H_{\mathrm{I}}, H_{\mathrm{II}}$ and $H_{\mathrm{III}}^{A}$ YangBaxter maps. The entwining maps of this method belongs to different subclasses than the $[2: 2]$ subclass of maps that the $H_{\mathrm{I}}, H_{\mathrm{II}}$ and $H_{\mathrm{III}}^{A}$ Yang-Baxter maps belongs to so they are not (Möb) ${ }^{2}$ equivalent to the latter. The outcomes of the second method are non-periodic ${ }^{7}$ entwining maps of subclass $[2: 2]$ associated with the whole $H$-list. The fact that the entwining maps which were presented in this Section preserve two invariants in separated variables, enable us to introduce appropriate potentials (as shown in $[44,45,56]$ ) to obtain integrable lattice equations. Actually we obtain integrable triplets of lattice equations (in some cases even correspondences). Note that integrable triplets of lattice equations were systematically derived in [13] and more recently in [33]. We plan to consider the integrable triplets of lattice equations derived from entwining maps, elsewhere.

In Section 6, we have proved that the transfer maps associated with the $H$ list of YangBaxter maps can be considered as the $(k-1)$-iteration of some maps of simpler form. As a consequence of this re-factorisation we have obtained $(k+1)$-point (see Proposition 5.6) and $k$ point (see Corollary 5.7) alternating recurrences which can be considered as alternating versions of some hierarchies of discrete Painlevé equations. Moreover, the autonomous versions of some of the $k$-point recurrences presented in Corollary 5.7, can be obtained by periodic reductions [58] (cf. [34]) of integrable lattice equations. Here we have obtained alternating $k$-point recurrences from Yang-Baxter maps without performing periodic reductions. Hence, our results might be compared/extended to the novel and independent frameworks introduced in [8, 10] and [38, 39], where by using symmetry arguments, integrable lattice equations and discrete Painlevé equations of 2 nd order were linked.

\footnotetext{
${ }^{7}$ The non-periodicity assures that these entwining maps are not (Möb) ${ }^{2}$ equivalent with the corresponding maps of the $H$-list.
} 


\section{A The $F$-list and the $\boldsymbol{H}$-list of quadrirational Yang-Baxter maps}

The Yang-Baxter maps $R$ of the $F$ and the $H$-list, explicitly read

$$
\begin{aligned}
& R: \mathbb{C P}^{1} \times \mathbb{C P}^{1} \ni(u, v) \mapsto(U, V) \in \mathbb{C P}^{1} \times \mathbb{C P}^{1}, \\
& U=\alpha v P, \quad V=\beta u P, \quad P=\frac{(1-\beta) u+\beta-\alpha+(\alpha-1) v}{\beta(1-\alpha) u+(\alpha-\beta) u v+\alpha(\beta-1) v}, \\
& U=\alpha v P, \quad V=\beta u P, \quad P=\frac{u-v+\beta-\alpha}{\beta u-\alpha v}, \\
& U=\frac{v}{\alpha} P, \quad V=\frac{u}{\beta} P, \quad P=\frac{\alpha u-\beta v}{u-v}, \\
& U=v P, \quad V=u P, \quad P=1+\frac{\beta-\alpha}{u-v}, \\
& U=v+P, \quad V=u+P, \quad P=\frac{\alpha-\beta}{u-v}, \\
& U=v Q, \quad V=u Q^{-1}, \quad Q=\frac{(\alpha-1) u v+(\beta-\alpha) u+\alpha(1-\beta)}{(\beta-1) u v+(\alpha-\beta) v+\beta(1-\alpha)}, \\
& U=v+Q, \quad V=u-Q, \quad Q=\frac{(\alpha-\beta) u v}{\beta u+\alpha v-\alpha \beta}, \\
& U=\frac{v}{\alpha} Q, \quad V=\frac{u}{\beta} Q, \quad Q=\frac{\alpha u+\beta v}{u+v}, \\
& U=v Q, \quad V=u Q Q^{-1}, \quad Q=\frac{1+\beta u v}{1+\alpha u v}, \\
& U=v-Q, \quad V=u+Q, \quad Q=\frac{\alpha-\beta}{u+v} .
\end{aligned}
$$

The maps above are depending on 2 complex parameters $\alpha, \beta$. The parameter $\alpha$ is associated with the first factor of the cartesian product $\mathbb{C P}^{1} \times \mathbb{C P}^{1}$, whereas the parameter $\beta$ with the second factor.

\section{Acknowledgements}

P.K. is grateful to Aristophanis Dimakis, Vassilios Papageorgiou and Anastasios Tongas, the organizers of the $4^{\text {th }}$ Workshop on Mathematical Physics-Integrable Systems (November $30-$ December 1, 2018, Department of Mathematics, University of Patras, Patras, Greece), where this work was finalized. Also, P.K. is grateful to James Atkinson, Allan Fordy, Nalini Joshi, Frank Nijhoff and to Pol Vanhaecke for very fruitful discussions on the subject, as well as to Maciej Nieszporski for the endless discussions towards the answer to the great question of integrable systems, Yang-Baxter maps and everything.

\section{References}

[1] Adler V.E., Recuttings of polygons, Funct. Anal. Appl. 27 (1993), 141-143.

[2] Adler V.E., Bäcklund transformation for the Krichever-Novikov equation, Int. Math. Res. Not. 1998 (1998), 1-4, arXiv:solv-int/9707015.

[3] Adler V.E., On a class of third order mappings with two rational invariants, arXiv:nlin.SI/0606056.

[4] Adler V.E., Bobenko A.I., Suris Yu.B., Classification of integrable equations on quad-graphs. The consistency approach, Comm. Math. Phys. 233 (2003), 513-543, arXiv:nlin.SI/0202024. 
[5] Adler V.E., Bobenko A.I., Suris Yu.B., Geometry of Yang-Baxter maps: pencils of conics and quadrirational mappings, Comm. Anal. Geom. 12 (2004), 967-1007, arXiv:math.QA/0307009.

[6] Adler V.E., Shabat A.B., Dressing chain for the acoustic spectral problem, Theoret. and Math. Phys. 149 (2006), 1324-1337, arXiv:nlin.SI/0604008.

[7] Adler V.E., Yamilov R.I., Explicit auto-transformations of integrable chains, J. Phys. A: Math. Gen. 27 (1994), 477-492.

[8] Atkinson J., Idempotent biquadratics, Yang-Baxter maps and birational representations of Coxeter groups, arXiv:1301.4613.

[9] Atkinson J., Nieszporski M., Multi-quadratic quad equations: integrable cases from a factorized-discriminant hypothesis, Int. Math. Res. Not. 2014 (2014), 4215-4240, arXiv:1204.0638.

[10] Atkinson J., Yamada Y., Quadrirational Yang-Baxter maps and the elliptic Cremona system, arXiv:1804.01794.

[11] Baxter R.J., Exactly solved models in statistical mechanics, Academic Press, Inc., London, 1982.

[12] Bazhanov V.V., Sergeev S.M., Yang-Baxter maps, discrete integrable equations and quantum groups, $N u$ clear Phys. B 926 (2018), 509-543, arXiv:1501.06984.

[13] Boll R., Classification of 3D consistent quad-equations, J. Nonlinear Math. Phys. 18 (2011), 337-365, arXiv:1009.4007.

[14] Bruschi M., Ragnisco O., Santini P.M., Tu G.Z., Integrable symplectic maps, Phys. D 49 (1991), $273-294$.

[15] Capel H.W., Sahadevan R., A new family of four-dimensional symplectic and integrable mappings, Phys. A 289 (2001), 86-106.

[16] Cresswell C., Joshi N., The discrete first, second and thirty-fourth Painlevé hierarchies, J. Phys. A: Math. Gen. 32 (1999), 655-669.

[17] Dimakis A., Müller-Hoissen F., Simplex and polygon equations, SIGMA 11 (2015), 042, 49 pages, arXiv:1409.7855.

[18] Dimakis A., Müller-Hoissen F., Matrix Kadomtsev-Petviashvili equation: tropical limit, Yang-Baxter and pentagon maps, Theoret. and Math. Phys. 196 (2018), 1164-1173, arXiv:1709.09848.

[19] Dimakis A., Müller-Hoissen F., Matrix KP: tropical limit and Yang-Baxter maps, Lett. Math. Phys. 109 (2019), 799-827, arXiv:1708.05694.

[20] Doliwa A., Non-commutative rational Yang-Baxter maps, Lett. Math. Phys. 104 (2014), 299-309, arXiv:1308.2824.

[21] Drinfel'd V.G., On some unsolved problems in quantum group theory, in Quantum Groups (Leningrad, 1990), Lecture Notes in Math., Vol. 1510, Springer, Berlin, 1992, 1-8.

[22] Duistermaat J.J., Discrete integrable systems. QRT maps and elliptic surfaces, Springer Monographs in Mathematics, Springer, New York, 2010.

[23] Etingof P., Geometric crystals and set-theoretical solutions to the quantum Yang-Baxter equation, Comm. Algebra 31 (2003), 1961-1973, arXiv:math.QA/0112278.

[24] Etingof P., Schedler T., Soloviev A., Set-theoretical solutions to the quantum Yang-Baxter equation, Duke Math. J. 100 (1999), 169-209, arXiv:math.QA/9801047.

[25] Evripidou C.A., Kassotakis P., Vanhaecke P., Integrable deformations of the Bogoyavlenskij-Itoh LotkaVolterra systems, Regul. Chaotic Dyn. 22 (2017), 721-739, arXiv:1709.06763.

[26] Fomin S., Zelevinsky A., Cluster algebras. I. Foundations, J. Amer. Math. Soc. 15 (2002), 497-529, arXiv:math.RT/0104151.

[27] Fomin S., Zelevinsky A., The Laurent phenomenon, Adv. in Appl. Math. 28 (2002), 119-144, arXiv:math.CO/0104241.

[28] Fordy A.P., Hone A., Discrete integrable systems and Poisson algebras from cluster maps, Comm. Math. Phys. 325 (2014), 527-584, arXiv:1207.6072.

[29] Fordy A.P., Kassotakis P., Multidimensional maps of QRT type, J. Phys. A: Math. Gen. 39 (2006), 1077310786.

[30] Fordy A.P., Kassotakis P., Integrable maps which preserve functions with symmetries, J. Phys. A: Math. Theor. 46 (2013), 205201, 12 pages, arXiv:1301.1927.

[31] Grahovski G.G., Konstantinou-Rizos S., Mikhailov A.V., Grassmann extensions of Yang-Baxter maps, J. Phys. A: Math. Theor. 49 (2016), 145202, 17 pages, arXiv:1510.06913. 
[32] Hay M., Hierarchies of nonlinear integrable $q$-difference equations from series of Lax pairs, J. Phys. A: Math. Theor. 40 (2007), 10457-10471.

[33] Hietarinta J., Search for CAC-integrable homogeneous quadratic triplets of quad equations and their classification by BT and Lax, J. Nonlinear Math. Phys. 26 (2019), 358-389, arXiv:1806.08511.

[34] Hietarinta J., Joshi N., Nijhoff F.W., Discrete systems and integrability, Cambridge Texts in Applied Mathematics, Cambridge University Press, Cambridge, 2016.

[35] Iatrou A., Higher dimensional integrable mappings, Phys. D 179 (2003), 229-253.

[36] Jimbo M. (Editor), Yang-Baxter equation in integrable systems, Adv. Ser. Math. Phys., Vol. 10, World Sci. Publ., Teaneck, NJ, 1989.

[37] Joshi N., Kassotakis P., Re-factorising a QRT map, arXiv:1906.00501.

[38] Joshi N., Nakazono N., Shi Y., Lattice equations arising from discrete Painlevé systems. I. $\left(A_{2}+A_{1}\right)^{(1)}$ and $\left(A_{1}+A_{1}^{\prime}\right)^{(1)}$ cases, J. Math. Phys. 56 (2015), 092705, 25 pages, arXiv:1401.7044.

[39] Joshi N., Nakazono N., Shi Y., Lattice equations arising from discrete Painlevé systems: II. $A_{4}^{(1)}$ case, J. Phys. A: Math. Theor. 49 (2016), 495201, 39 pages, arXiv:1603.09414.

[40] Kajiwara K., Noumi M., Yamada Y., Discrete dynamical systems with $W\left(A_{m-1}^{(1)} \times A_{n-1}^{(1)}\right)$ symmetry, Lett. Math. Phys. 60 (2002), 211-219, arXiv:nlin.SI/0106029.

[41] Kashaev R.M., On discrete three-dimensional equations associated with the local Yang-Baxter relation, Lett. Math. Phys. 38 (1996), 389-397, arXiv:solv-int/9512005.

[42] Kashaev R.M., Korepanov I.G., Sergeev S.M., Functional tetrahedron equation, Theoret. and Math. Phys. 117 (1998), 1402-1413, arXiv:solv-int/9801015.

[43] Kassotakis P., The construction of discrete dynamical system, Ph.D. Thesis, University of Leeds, 2006.

[44] Kassotakis P., Nieszporski M., Families of integrable equations, SIGMA 7 (2011), 100,14 pages, arXiv:1106.0636.

[45] Kassotakis P., Nieszporski M., On non-multiaffine consistent around the cube lattice equations, Phys. Lett. A 376 (2012), 3135-3140, arXiv:1106.0435.

[46] Kassotakis P., Nieszporski M., $2^{n}$-rational maps, J. Phys. A: Math. Theor. 50 (2017), 21LT01, 9 pages, arXiv:1512.00771.

[47] Kassotakis P., Nieszporski M., Difference systems in bond and face variables and non-potential versions of discrete integrable systems, J. Phys. A: Math. Theor. 51 (2018), 385203, 21 pages, arXiv:1710.11111.

[48] Konstantinou-Rizos S., Mikhailov A.V., Darboux transformations, finite reduction groups and related YangBaxter maps, J. Phys. A: Math. Theor. 46 (2013), 425201, 16 pages.

[49] Korepanov I.G., Algebraic integrable dynamical systems, 2+1-dimensional models in wholly discrete spacetime, and inhomogeneous models in 2-dimensional statistical physics, arXiv:solv-int/9506003.

[50] Kouloukas T.E., Relativistic collisions as Yang-Baxter maps, Phys. Lett. A 381 (2017), 3445-3449, arXiv:1706.06361.

[51] Kouloukas T.E., Papageorgiou V.G., Entwining Yang-Baxter maps and integrable lattices, in Algebra, Geometry and Mathematical Physics, Banach Center Publ., Vol. 93, Polish Acad. Sci. Inst. Math., Warsaw, 2011, 163-175.

[52] Maeda S., Completely integrable symplectic mapping, Proc. Japan Acad. Ser. A Math. Sci. 63 (1987), $198-200$.

[53] Maillet J.-M., Nijhoff F., Integrability for multidimensional lattice models, Phys. Lett. B 224 (1989), 389396.

[54] Maillet J.-M., Nijhoff F., The tetrahedron equation and the four-simplex equation, Phys. Lett. A 134 (1989), 221-228.

[55] McLachlan R.I., Quispel G.R.W., Generating functions for dynamical systems with symmetries, integrals, and differential invariants, Phys. D 112 (1998), 298-309.

[56] Nieszporski M., Kassotakis P., Systems of difference equations on a vector valued function that admit a 3D vector space of scalar potentials, in preparation.

[57] Noumi M., Yamada Y., Affine Weyl groups, discrete dynamical systems and Painlevé equations, Comm. Math. Phys. 199 (1998), 281-295, arXiv:math.QA/9804132. 
[58] Papageorgiou V.G., Nijhoff F.W., Capel H.W., Integrable mappings and nonlinear integrable lattice equations, Phys. Lett. A 147 (1990), 106-114.

[59] Papageorgiou V.G., Suris Yu.B., Tongas A.G., Veselov A.P., On quadrirational Yang-Baxter maps, SIGMA 6 (2010), 033, 9 pages, arXiv:0911.2895.

[60] Papageorgiou V.G., Tongas A.G., Veselov A.P., Yang-Baxter maps and symmetries of integrable equations on quad-graphs, J. Math. Phys. 47 (2006), 083502, 16 pages, arXiv:math.QA/0605206.

[61] Quispel G.R.W., Roberts J.A.G., Thompson C.J., Integrable mappings and soliton equations, Phys. Lett. A 126 (1988), 419-421.

[62] Roberts J.A.G., Quispel G.R.W., Creating and relating three-dimensional integrable maps, J. Phys. A: Math. Gen. 39 (2006), L605-L615.

[63] Sakai H., Rational surfaces associated with affine root systems and geometry of the Painlevé equations, Comm. Math. Phys. 220 (2001), 165-229.

[64] Sergeev S.M., Solutions of the functional tetrahedron equation connected with the local Yang-Baxter equation for the ferro-electric condition, Lett. Math. Phys. 45 (1998), 113-119, arXiv:solv-int/9709006.

[65] Sklyanin E.K., Classical limits of SU(2)-invariant solutions of the Yang-Baxter equation, J. Sov. Math. 40 (1988), 93-107.

[66] Suris Yu.B., Veselov A.P., Lax matrices for Yang-Baxter maps, J. Nonlinear Math. Phys. 10 (2003), suppl. 2, 223-230, arXiv:math.QA/0304122.

[67] Tsuda T., Integrable mappings via rational elliptic surfaces, J. Phys. A: Math. Gen. 37 (2004), 2721-2730.

[68] Veselov A.P., Integrable maps, Russian Math. Surveys 46 (1991), no. 5, 1-51.

[69] Veselov A.P., Yang-Baxter maps and integrable dynamics, Phys. Lett. A 314 (2003), 214-221, arXiv:math.QA/0205335.

[70] Veselov A.P., Yang-Baxter maps: dynamical point of view, in Combinatorial Aspect of Integrable Systems, MSJ Mem., Vol. 17, Math. Soc. Japan, Tokyo, 2007, 145-167.

[71] Veselov A.P., Shabat A.B., Dressing chains and the spectral theory of the Schrödinger operatorr, Funct. Anal. Appl. 27 (1993), 81-96.

[72] Viallet C.M., Integrable lattice maps: $Q_{\mathrm{V}}$, a rational version of $Q_{4}$, Glasg. Math. J. 51 (2009), 157-163, arXiv:0802.0294.

[73] Yang C.N., Some exact results for the many-body problem in one dimension with repulsive delta-function interaction, Phys. Rev. Lett. 19 (1967), 1312-1315. 